\title{
Article \\ Expression of CCRL2 Inhibits Tumor Growth by Concentrating Chemerin and Inhibiting Neoangiogenesis
}

\author{
Diana Al Delbany ${ }^{1}\left(\right.$, , Virginie Robert ${ }^{1,2}$, Ingrid Dubois-Vedrenne ${ }^{1,3}$, Annalisa Del Prete ${ }^{4,5}($, \\ Maxime Vernimmen ${ }^{1}$, Ayoub Radi ${ }^{1}$, Anne Lefort ${ }^{1}$, Frédérick Libert ${ }^{1}$, Valérie Wittamer ${ }^{1}{ }^{1}$, Silvano Sozzani ${ }^{6,7}$ \\ and Marc Parmentier ${ }^{1, *}$ \\ 1 I.R.I.B.H.M and Welbio, Campus Erasme, Université Libre de Bruxelles, 808 Route de Lennik, \\ B-1070 Brussels, Belgium; delbanidiana@outlook.com (D.A.D.); virginie.gavioli@evotec.com (V.R.); \\ gridou24@hotmail.fr (I.D.-V.); maximevernimmen@hotmail.com (M.V.); ayoub.radi@outlook.com (A.R.); \\ anne.lefort@ulb.be (A.L.); frederick.libert@ulb.be (F.L.); valerie.wittamer@ulb.be (V.W.) \\ 2 Evotec SAS, 195 Route d'Espagne, 31036 Toulouse, France \\ 3 Institute for Medical Immunology, Université Libre de Bruxelles, Rue Adrienne Bolland 8, \\ 6041 Gosselies, Belgium \\ 4 Department of Molecular and Translational Medicine, University of Brescia, Viale Europa 11, \\ 25123 Brescia, Italy; annalisa.delprete@unibs.it \\ 5 Humanitas Clinical and Research Center-IRCCS, Via Manzoni 56, 20089 Rozzano, Italy \\ 6 Laboratory Affiliated to Istituto Pasteur Italia-Fondazione Cenci Bolognetti, Department of Molecular \\ Medicine, Sapienza University of Rome, 00185 Rome, Italy; silvano.sozzani@uniroma1.it \\ 7 IRCCS Neuromed, 86077 Pozzilli, Italy \\ * Correspondence: marc.parmentier@ulb.be; Tel.: +32-2-55541-71
}

Citation: Al Delbany, D.; Robert, V.; Dubois-Vedrenne, I.; Del Prete, A.; Vernimmen, M.; Radi, A.; Lefort, A.; Libert, F.; Wittamer, V.; Sozzani, S.; et al. Expression of CCRL2 Inhibits Tumor Growth by Concentrating Chemerin and Inhibiting Neoangiogenesis. Cancers 2021, 13, 5000. https://doi.org/10.3390/ cancers 13195000

Academic Editors: Oliver Treeck and Christa Büchler

Received: 19 August 2021

Accepted: 1 October 2021

Published: 5 October 2021

Publisher's Note: MDPI stays neutral with regard to jurisdictional claims in published maps and institutional affiliations.

Copyright: () 2021 by the authors. Licensee MDPI, Basel, Switzerland. This article is an open access article distributed under the terms and conditions of the Creative Commons Attribution (CC BY) license (https:// creativecommons.org/licenses/by/ $4.0 /)$.
Simple Summary: Chemerin is a multifunctional protein regulating inflammation, immune responses, and metabolism. It was also shown to display anti-tumoral properties in various cancer models. CMKLR1 is the main functional receptor of chemerin. C-C motif chemokine receptor-like 2 (CCRL2) is another receptor binding chemerin with high affinity but failing to signal through any known signaling pathway. CCRL2 is strongly upregulated by inflammatory signals and was shown to regulate inflammatory reactions in diverse pathological conditions. Expression of CCRL2 was described in many types of human tumors such as melanoma, neuroblastoma, prostate, breast, and gastric cancer. However, its functional role in cancer has not been studied much so far. We investigate in this study how CCRL2 expression can influence the distribution of chemerin and thereby its biological activity in different tumoral contexts.

Abstract: CCRL2 belongs to the G protein-coupled receptor family and is one of the three chemerin receptors. It is considered as a non-signaling receptor, presenting chemerin to cells expressing the functional chemerin receptor ChemR23/CMKLR1 and possibly GPR1. In the present work, we investigate the role played by CCRL2 in mouse cancer models. Loss of function of Ccrl2 accelerated the development of papillomas in a chemical model of skin carcinogenesis (DMBA/TPA), whereas the growth of B16 and LLC tumor cell grafts was delayed. Delayed tumor growth was also observed when B16 and LLC cells overexpress CCRL2, while knockout of Ccrl2 in tumor cells reversed the consequences of Ccrl2 knockout in the host. The phenotypes associated with CCRL2 gain or loss of function were largely abrogated by knocking out the chemerin or Cmklr1 genes. Cells harboring CCRL2 could concentrate bioactive chemerin and promote the activation of CMKLR1-expressing cells. A reduction of neoangiogenesis was observed in tumor grafts expressing CCRL2, mimicking the phenotype of chemerin-expressing tumors. This study demonstrates that CCRL2 shares functional similarities with the family of atypical chemokine receptors (ACKRs). Its expression by tumor cells can significantly tune the effects of the chemerin/CMKLR1 system and act as a negative regulator of tumorigenesis.

Keywords: chemerin; ChemR23; CMKLR1; Rarres2; tumor angiogenesis 


\section{Introduction}

Cancer is the result of a multi-step process requiring the accumulation of mutations in different oncogenes and tumor suppressor genes in the progeny of a single cell [1]. The tumor microenvironment is well known to influence many aspects of cancer development. Various leukocyte populations contribute largely to this microenvironment, and leukocyte chemoattractant molecules, including chemokines, play therefore key roles in cancer progression, more particularly by tuning cancer-related inflammation [2]. The expression of chemoattractant molecules and their receptors is partly caused by genetic events that contribute to neoplastic transformation and play an important role in chronic inflammatory conditions that predispose to cancer. Components of the leukocyte trafficking system affect multiple aspects of tumor progression, including leukocyte recruitment, tumor cell proliferation and survival, neoangiogenesis, epithelial to mesenchymal transition, local invasion, and metastasis.

Chemerin, encoded by the retinoic acid receptor responder 2 (RARRES2) gene, also known as tazarotene-induced gene 2 (TIG2), is a small secreted protein endowed with chemotactic activity for monocytes/macrophages, myeloid and plasmacytoid dendritic cells, and NK cells [3]. Chemerin binds to three G protein-coupled receptors (GPCR): CMKLR1 (also known as ChemR23 or chemerin 1 ), GPR1 (or chemerin 2 ), and CCRL2 [4]. A large number of studies have investigated the role of chemerin in tumor biology and the potential value of chemerin as a diagnostic or prognostic biomarker in various cancer types $[5,6]$. Downregulation of chemerin expression was described in many cancers, including non-small-cell lung carcinoma (NSCLC) [7], squamous cell carcinoma of the skin [6], and melanoma [8], and such downregulation was often associated with poor clinical outcomes. In line with this observation, chemerin expression by cancer cells was shown to delay tumor growth and progression in various mouse models. Immune cell recruitment to the tumor microenvironment, direct effects on cancer cells, and a reduction of tumoral angiogenesis were proposed as mechanisms $[7,9,10]$.

Atypical chemokine receptors (ACKR) have emerged as new regulators of the chemokine system, given their capacity to control chemokine bioavailability and the signaling of functional receptors $[11,12]$. ACKRs were shown to play essential roles in tumor biology in nearly every key step of progression from tumor initiation to metastasis, including cancer cell proliferation, the recruitment of tumor-associated leukocytes, tumoral angiogenesis, adherence to endothelium and extravasation, and defense against host immune responses [13]. ACKRs influence the behavior of malignant cells as well as of various stromal cells. ACKRs can function in a cell-autonomous manner, affecting the response of the cells in which they are expressed, or indirectly by influencing chemokine receptor signaling in other cells [13]. CCRL2 is structurally related to chemokine receptors CCR1 to 5, but identified as an atypical receptor for chemerin [14,15]. Both human and mouse CCRL2 lack the consensus "DRYLAIV" motif involved in the coupling to heterotrimeric G proteins, which led to the hypothesis that CCRL2 does not function as a classical chemoattractant receptor. CCRL2 binds chemerin with high affinity and shows a low level of constitutive endocytosis that is not enhanced following chemerin binding. CCRL2 is therefore considered as a chemerin binding site, controlling the bioavailability of the protein by increasing its local concentration and presenting it to other cells expressing CMKLR1, the main functional receptor of chemerin [14]. Different cell types express CCRL2 in humans and mice, including macrophages, neutrophils, dendritic cells, microglia, and endothelial cells [16]. CCRL2 expression by tumor cells was also described in several human cancer types, including glioblastoma [17], breast [18], and colorectal cancer [19]. However, the functional role of CCRL2 in cancer has been investigated in few studies so far. A recent study showed that CCRL2 played an anti-tumoral role by contributing to the recruitment of NK cells to tumors in a mouse model of lung cancer [20].

In the present work, we investigate the impact of CCRL2 expression by the host and tumor cells on the chemerin-CMKLR1 axis and its consequences on tumor progression. For this purpose, we used a chemical model of skin carcinogenesis and tumoral cell lines 
overexpressing or knocked out for Ccrl2, as well as various mouse genetic models affecting elements of the chemerin system. We show that expression of CCRL2 by tumor cells enhances the local activity of chemerin, which inhibits neoangiogenesis and results in a reduction of tumor growth and progression.

\section{Materials and Methods}

\subsection{Mice}

C57BL/6J and NOD/SCID mice were purchased from Janvier. The Ccrl2 [21] and Cmklr1 [22] knockout mice were described previously. The Gpr1 knockout line (B6NDen;

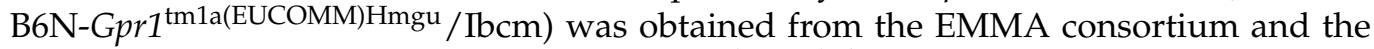
chemerin knockout line (C57BL/6N-Rarres2 $2^{\operatorname{tm} 1(K O M P) V l c g} / \mathrm{MbpMmucd)}$ from the Mutant Mouse Resource and Research Center at the University of California at Davis. Mice expressing bioactive chemerin under control of the keratin K5 promoter (K5-chemerin) were described and characterized elsewhere [23]. All strains were backcrossed into the C57BL/6J background for more than 20 generations. Mice were maintained in a specific pathogen-free environment and, except otherwise stated, used between 6 and 12 weeks of age. All animal experiments were conducted following European guidelines and local regulations and approved by the ethics committee (Commission d'Ethique du Bien-Etre Animal, CEBEA) of the ULB Medical School. All efforts were made to minimize suffering.

\subsection{Cell Lines}

Murine B16-F0 melanoma and Lewis lung carcinoma (LLC) cell lines were purchased from the American Type Culture Collection (ATCC). Cells were grown in RPMI-1640 (Life Technologies, Merelbelke Belgium) supplemented with 10\% fetal bovine serum (Gibco, Paisley, UK), 1\% sodium pyruvate (Gibco), $100 \mathrm{U} / \mathrm{mL}$ penicillin, and $100 \mu \mathrm{g} / \mathrm{mL}$ streptomycin (Invitrogen, Carlsbad, CA, USA). The cells were cultured at $37^{\circ} \mathrm{C}$ in a humidified atmosphere containing $5 \% \mathrm{CO}_{2}$. The various clones were regularly tested negative for mycoplasma infection.

\subsection{Knockout of Ccrl2 in Tumoral Cell Lines}

LLC and B16 clones knocked out for Ccrl2 were generated using an approach that combines a mutant Cas9 nickase and paired CRISPR guide RNAs (sgRNA) targeting the region immediately following the AUG start codon in the second and only coding exon of the Ccrl2 gene. The sgRNAs, designed using the CRISPR design tool available on the Zhang Lab website (http: / / crispr.mit.edu) (accessed on 14 October 2016) were 5'-GGATTAGAATCTGCTGATGGACC-3' (Guide A) and 5'-GGCCTTGAACCAGGCCGGGTGAC-3' (Guide B) and were cloned into the pSpCas9n (BB)-2A-GFP (PX461) vector (Addgene, Teddington, UK). Following sequencing, the plasmids were cotransfected in LLC and B16 cells using Lipofectamine 2000 (ThermoFisher, Waltham, MA, USA) according to the manufacturer's instructions. Three days after transfection, single GFP-positive cells were sorted into 96-well plates using a FACSAria cell sorter (BD Biosciences), and the clonal growth was controlled for a week after sorting. The clones were screened by PCR using 5'-TGTCGGATGGAGGGGAATCA-3' as the forward primer and $5^{\prime}$ CCAAGATAAACACCGCCAGC $-3^{\prime}$ as the reverse primer flanking the sgRNA target sites, and a DNA heteroduplex mobility assay on polyacrylamide gels was used to detect small deletions or insertions ( 1 to $4 \mathrm{bp}$ ). The presence of frameshifts and premature stop codons in the two alleles was characterized by cloning the PCR products and sequencing a minimum of 5 clones per cell line.

\subsection{Overexpression of CCRL2 in Tumoral Cell Lines}

LLC and B16 stably overexpressing CCRL2 were generated by transfecting cells with a bicistronic expression plasmid ( $\mathrm{pCDNeo}$ ) containing a codon-optimized version of the mouse Ccrl2 cDNA and the neomycin (G418) resistance gene driven by an EF-1 $\alpha$ promoter. Cells were seeded into 24-well plates containing serum-free medium and transfected using the X-tremeGENE Transfection reagent (Roche, Mannheim, Germany) according 
to the manufacturer's instructions. Two days after transfection, the cells were re-seeded onto Petri dishes in a medium containing $800 \mu \mathrm{g} / \mathrm{mL}$ G418 (Invivogen), and individual G418-resistant clones were selected. The level of CCRL2 expression by B16-CCRL2 and LLCCCRL2 clones was determined by PCR using 5'-ACGAGCCCAGAATGGAGAGA-3 ${ }^{\prime}$ as the forward primer and $5^{\prime}$-GCTTGTGCAGGTCGTACTGT- $3^{\prime}$ as the reverse primer (specific to the codon-optimized Ccrl2 sequence), and by FACS analysis using a phycoerythrin (PE)-conjugated anti-mouse CCRL2 monoclonal antibody (clone BZ2E3, BD Pharmingen, San Diego, CA, USA).

\subsection{Proliferation Assays}

The proliferation rate of the various B16 and LLC clones was determined by two procedures. Cells were seeded in 96-well plates (1000 cells/well in $100 \mu \mathrm{L}$ of growth medium) and cultured for 1 to 4 days. Every day, the medium was removed from part of the wells, and the cells were incubated for $4 \mathrm{~h}$ at $37^{\circ} \mathrm{C}$ with $10 \mu \mathrm{L}$ of a $5 \mathrm{mg} / \mathrm{mL} 3-(4,5-$ dimethylthiazol-2-yl)-2,5 diphenyl tetrazolium bromide (MTT, Sigma-Aldrich, Overijse, Belgium) solution. DMSO $(100 \mu \mathrm{L})$ was added and the wells gently shaken until dissolution of the formazan crystals. The absorbance at $570 \mathrm{~nm}$ was recorded in a microplate reader (BioRad, Temse, Belgium), and cell proliferation was inferred by dividing the absorbance at day $\mathrm{X}$ by the absorbance at day 1 . The experiment was performed three times with sextuplicate wells.

B16 and LLC cells were also seeded in 6-well plates at densities of respectively 0.25 and $0.3 \times 10^{6}$ cells per well and cultured for 3 days. The cells were collected every day from part of the wells and counted using an EVE automatic cell counter (NanoEnTek). Cell proliferation was determined by dividing the cell number at day $X$ by the number of cells seeded at day 0 . The doubling time of the clones was also determined regularly as described previously [24]. Briefly, after harvesting the cells, $10 \mu \mathrm{L}$ of the suspension was stained with trypan blue, and live cells were counted in the EVE counter. The ratio was calculated since the previous passage and the doubling time measured twice a week for each cell line.

\subsection{Chemerin Presentation Assay}

The ability of cells expressing CCRL2 to bind recombinant mouse chemerin and present it to cells expressing CMKLR1 was assessed using an aequorin-based calcium mobilization assay [25-27]. CHO-K1 cells co-expressing mouse CMKLR1, apoaequorin and $\mathrm{G} \alpha 16$, and control CHO-K1 cells expressing only apoaequorin and G $\alpha 16$, were collected from plates with phosphate-buffered saline (PBS) supplemented with 5 mM EDTA, pelleted, resuspended at a density of $5 \times 10^{6}$ cells $/ \mathrm{mL}$ in aequorin buffer (DMEM/Ham's F12 containing $0.5 \%$ BSA), and incubated with $5 \mu \mathrm{M}$ coelenterazine $\mathrm{H}$ (Promega) for $4 \mathrm{~h}$ at room temperature under gentle agitation in the dark. Cells were then diluted 10-fold and incubated for one hour. B16, B16-CCRL2, and B16-Crispr ${ }^{C} c r l 2$ cells were grown to confluence in 96-well microplates and starved for $24 \mathrm{~h}$ in a serum-free medium. The next day, the cells were incubated at $37{ }^{\circ} \mathrm{C}$ for $2 \mathrm{~h}$ with $5 \mathrm{nM}$ recombinant mouse chemerin (R\&D Systems) in PBS containing 0.1\% BSA. The cells were then washed with PBS/BSA to remove unbound chemerin, and $50 \mu \mathrm{L}$ of aequorin buffer were added to the wells. $\mathrm{CHO}$ cells expressing or not CMKLR1 $(25,000$ cells in $50 \mu \mathrm{L})$ were added to wells containing B16, B16-CCRL2, or B16-CriprCcrl2 cells preincubated with chemerin, and luminescence was recorded for $20 \mathrm{~s}$ in a Packard luminometer. Results (as luminescence units) were normalized to the response to $10 \mu \mathrm{M}$ ATP.

\subsection{GFP-Chemerin Binding Assay}

A recombinant baculovirus containing a GFP-chemerin fusion was constructed by the Bac-to-Bac system (Invitrogen). The GFP-chemerin fusion was cloned into the pFastBac vector (Invitrogen) between restriction sites $\mathrm{NdeI}$ and $\mathrm{Not} \mathrm{I}$, and the plasmid transferred into DH10Bac E. coli cells in order to generate the recombinant bacmid. SF9 cells were 
transfected with the bacmid to generate the viral stock. For the GFP-chemerin fusion protein production, SF9 cells were infected with the viral stock, and the supernatant was collected $72 \mathrm{~h}$ later. The fusion protein was purified on a HiTrap heparin column (Cytiva 17-0406-01), and the concentration of recombinant protein was determined by ELISA and Western blotting, using recombinant chemerin as a reference control. Tumor cells were grown on polylysine-treated 24-well glass bottom sensoPlates (Greiner, 662892, Kremsmünster, Austria) to $60-80 \%$ confluence. Cells were incubated in serum-free media for $24 \mathrm{~h}$, then with $10 \mathrm{nM}$ of GFP-chemerin for $2 \mathrm{~h}$ at $37^{\circ} \mathrm{C}$. The ligand was removed, and the cells were fixed for $20 \mathrm{~min}$ with $4 \%$ paraformaldehyde and washed three times with PBS. They were further incubated overnight at $4{ }^{\circ} \mathrm{C}$ with a rabbit anti-GFP antibody (Abcam, $\mathrm{Ab6550)}$, then for $2 \mathrm{~h}$ in the dark at room temperature with an Alexa Fluor 488-conjugated goat anti-rabbit IgG (1:500, Invitrogen). Nuclei were stained with Hoechst 33,342 (1:4000, Life Technologies). Images were acquired using a Zeiss AxoImager Z1 microscope (Carl Zeiss, Jena, Germany) and analyzed with the ImageJ software.

\subsection{Tumor Models}

B16-F0 or LLC cells $\left(10^{6}\right)$ were grafted subcutaneously into the back of syngeneic C57BL/6J mice. The size of the resulting tumors was monitored every other day from day 3 with a caliper, and the tumor volume was estimated by the formula $\mathrm{V}=$ (length $\times$ width $\left.^{2}\right) / 2$. At the end of the experiment, mice were killed by cervical dislocation, and tumors were collected for further analysis.

The DMBA/TPA two-stage chemical carcinogenesis model was performed as previously described [28], using eight-week-old mice of the C57BL/6J background. Mice were treated during the first and seventh week with 9,10-dimethyl-1,2-benzanthracene (DMBA, Sigma, $50 \mu \mathrm{g}$ in $200 \mu \mathrm{L}$ acetone) applied on shaved skin three times at two-day intervals, and with 12-O-tetradecanoyl phorbol-13-acetate (TPA, Sigma, $4 \mu \mathrm{g}$ in $200 \mu \mathrm{L}$ acetone), applied twice a week from weeks 2 to 6 and from week 8 onwards. The number and size of tumors were recorded every other week.

\subsection{Flow Cytometry Analysis}

Tumors were cut into small fragments (about $1 \mathrm{~mm}^{3}$ ) and digested for $1 \mathrm{~h} 30 \mathrm{~min}$ at $37{ }^{\circ} \mathrm{C}$ on a rocking plate in HBSS medium containing $5 \%$ fetal bovine serum, $1 \mathrm{mg} / \mathrm{mL}$ collagenase D (Roche), and $200 \mathrm{U} / \mathrm{mL}$ DNase I (Roche). After addition of $5 \mathrm{mM}$ EDTA to block collagenase D activity, the cell suspension was rinsed with PBS and tissue debris eliminated by passing through a 70- $\mu$ m nylon mesh. Single-cell suspensions were incubated for $20 \mathrm{~min}$ at $4{ }^{\circ} \mathrm{C}$ with anti-CD16/CD32 Fc Block (eBioscience, San Diego, CA, USA) in PBS containing 1\% FCS, $1 \mathrm{mM}$ EDTA, and 0.1\% NaN3 (FACS buffer) and stained for 30 min at $4{ }^{\circ} \mathrm{C}$ with a mixture of antibodies in FACS buffer. Antibodies recognizing CD45 (47-0451 and 17-0451-83), NK1.1 (12-5941-82), F4/80 (BM8), and CD3 (17-0032-82) were from eBioscience, and CD11b (550993 and 553311), CD11c (550261), Gr1 (552093), and B220 (RA3-6B2) from BD Pharmingen. Tumoral cell lines were also stained with an anti-mouse CCRL2 (clone BZ2E3, BD Pharmingen). Flow cytometry analysis was performed on an LSRFortessa instrument (BD Biosciences, Franklin Lakes, NJ, USA) and analyzed using the FlowJo software.

\subsection{Histological Procedures}

Tissues were embedded in OCT (Tissue Tek, Sakura, Berchem, d sectioned at $8 \mu \mathrm{m}$ using a Leica cryostat. Sections were post-fixed in acetone for $10 \mathrm{~min}$ at $4{ }^{\circ} \mathrm{C}$. For immunofluorescence analysis, the sections were blocked with $1 \%$ rat serum, incubated overnight at $4{ }^{\circ} \mathrm{C}$ with PE-conjugated mouse anti-CD31 and APC-conjugated mouse anti- $\alpha$ SMA (eBioscience), nuclei were stained with Hoechst 33,342 (1:4000, Life Technologies), and slides were mounted in DAKO mounting medium supplemented with 2.5\% 4-diazabicyclo [2.2.2] octane (DABCO, Sigma). Images were acquired using a Zeiss Axio Zoom V16 microscope (Carl Zeiss) and analyzed with the ImageJ software. 


\subsection{1. $R T-q P C R$}

Tumors and tumor cell lines were lysed in TRIzol (Life Technologies), and mRNAs were extracted using the RNAeasy Minikit (Qiagen Benelux, Antwerp, Belgium). RNA samples $(1 \mu \mathrm{g})$ were treated with DNase I (Invitrogen) and transcribed into cDNA using oligo(dT) and SuperscriptIII (Invitrogen). Reactions were performed in a final volume of $20 \mu \mathrm{L}$ using the Power SYBR Green PCR Master Mix (ThermoFisher) on a 7500 Fast thermocycler (Applied Biosystems, Waltham, MA, USA). Glyceraldehyde-3-phosphate dehydrogenase (GAPDH) was used as reference. Relative mRNA levels were calculated according to formula $2^{-\Delta C_{T}}$, in which $\Delta C_{T}=C_{T}$ (target gene) $-C_{T}(G A P D H)$. The primers used were $5^{\prime}-$ AAGCTCCAGCAGACCAACTG-3' (forward) and $5^{\prime}$-TTTACCCTTGGGGTCCATTT-3' (reverse) for chemerin, $5^{\prime}$-CCATGTGCAAGATCAGCAAC-3' (forward) and $5^{\prime}$-GCAGGAAGA CGCTGGTGTA-3' (reverse) for CMKLR1, 5'-GAGCAAGGACAGCCTCCGAT-3' (forward) and $5^{\prime}$-CCACTGTTGTCCAGGTAGTCG-3' (reverse) for CCRL2, 5'-GCTGCTGCTTATGGG CTTCTC-3' (forward) and 5'-TCACTGGGCAGTTTCTAGGAG-3' (reverse) for GPR1 and 5'-AAGGGCTCATGACCACAGTC-3' (forward) and 5'-CAGGGATGATGTTCTGGGCA-3' (reverse) for GAPDH.

\subsection{RNA Sequencing and Transcriptome Analysis}

Tumors were lysed in TRIzol, and total RNA extracted with the RNAeasy micro kit (Qiagen) following the manufacturer's instructions. RNA quality was checked on a Bioanalyzer 2100 (Agilent Technologies, Machelen, Belgium). Indexed cDNA libraries were obtained using the TruSeq RNA sample preparation kit (Illumina, San Diego, CA, USA). The multiplexed libraries (10 pM) were loaded on flow cells, and sequences were produced using a HiSeq PE Cluster Kit v4 and TruSeq SBS Kit v3-HS on a Hiseq 1500 (Illumina). Approximately 35 million paired reads per sample were mapped against the mouse reference genome (GRCm38.p4/mm10, ftp.Ensembl.org) using the STAR software to generate read alignments for each sample. Counts were obtained using HTSeq and differential gene expression was calculated on the Degust website (http://www. vicbioinformatics.com/degust/) (accessed on 13 September 2016) using EdgeR. The data have been deposited in NCBI's Gene Expression Omnibus and are accessible through GEO Series accession number GSE183914. Gene signatures were analyzed on the Gorilla (http:/ / cbl-gorilla.cs.technion.ac.il) (accessed on 4 October 2016) and GSEA (http:/ / software.broadinstitute.org/gsea/index.jsp) (accessed on 20 October 2016) websites [29,30].

\subsection{Statistical Analyses}

Statistical analyses and data graphing were performed using Prism 6 (GraphPad Software, version 6.01, San Diego, CA, USA). Statistical significance was calculated by the Mann-Whitney test for comparisons between two groups, or by one-way ANOVA followed by Tukey-Kramer multiple comparisons test for more than two groups, as indicated in the figure legends. $p$-values $<0.05$ were considered significant.

\section{Results}

3.1. CCRL2 Deficiency Accelerates Tumor Progression in a Chemical Model of Skin Carcinogenesis and Delays Tumor Growth in Graft Models

Ccrl2 KO mice and their WT controls were subjected to a two-stage chemical model of skin carcinogenesis (DMBA/TPA) as previously described [23]. Ccrl2 KO mice developed the first papillomas by week 11 of the treatment, while they appeared only at week 15 in the control group (Figure 1A). Ccrl2 KO mice also exhibited a larger number of tumors and faster tumor progression compared to control mice (Figure 1A,B). Indeed, the proportion of large papillomas (>3 mm) and carcinomas reached $25.4 \%$ in Ccrl $2 \mathrm{KO}$ mice versus $18.9 \%$ in control mice by the end of the experiment (Figure 1B). We also investigated the function of CCRL2 in tumor graft models. Following the graft of B16-F0 melanoma or Lewis lung carcinoma cells under the back skin, Ccrl2 $\mathrm{KO}$ mice developed smaller tumors than WT mice (Figure 1C-F). These contrasting results in different tumor models appeared at first 
puzzling, but it was reasoned that in graft models, the tumor cell lines are not knocked out for Ccrl2. It is well known that CCRL2 is upregulated in inflammatory conditions, which are frequently present in the tumor microenvironment, and expression of this receptor by tumor cells was reported in several human cancer types. We therefore tested the presence of $\mathrm{C}$ crl2 transcripts by qRT-PCR and confirmed the expression of the receptor in LLC and B16 tumors collected from WT and Ccrl2 KO mice (data not shown). These results were confirmed in RNAseq data obtained on these tumors (Figure 1G). The expression level was somewhat higher in LLC tumors than in B16 tumors and unaffected by the genotype of the mice (Ccrl2 KO or WT), demonstrating that the transcripts derive primarily from tumor cells and less from stromal cells of the microenvironment, such as leucocytes or endothelial cells.

A

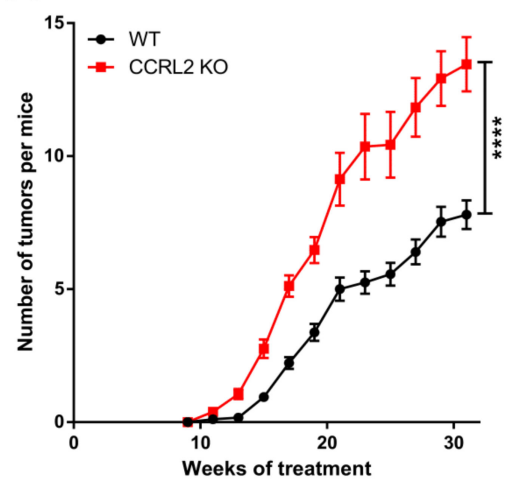

C

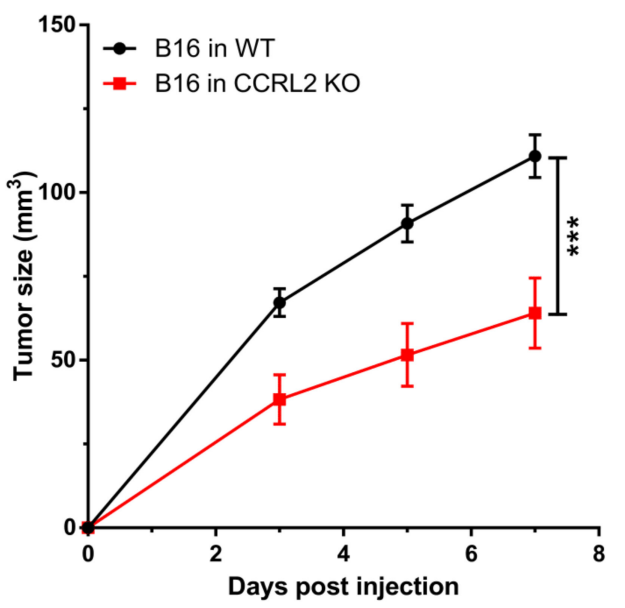

B

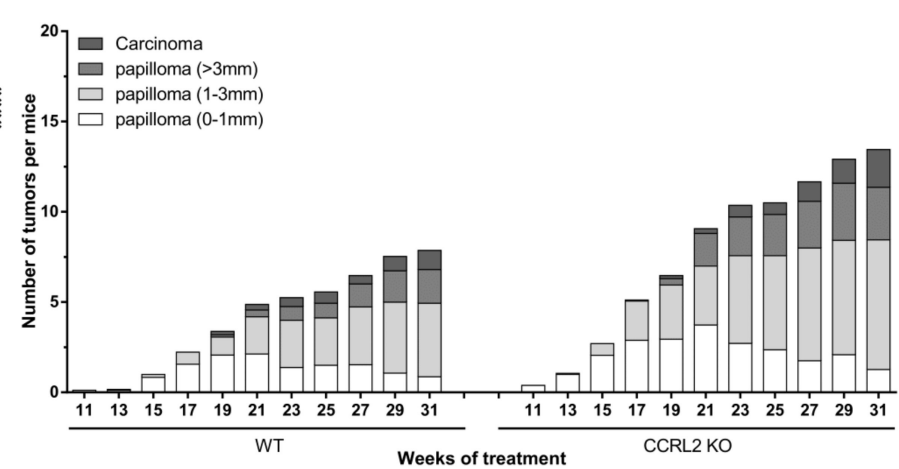

D

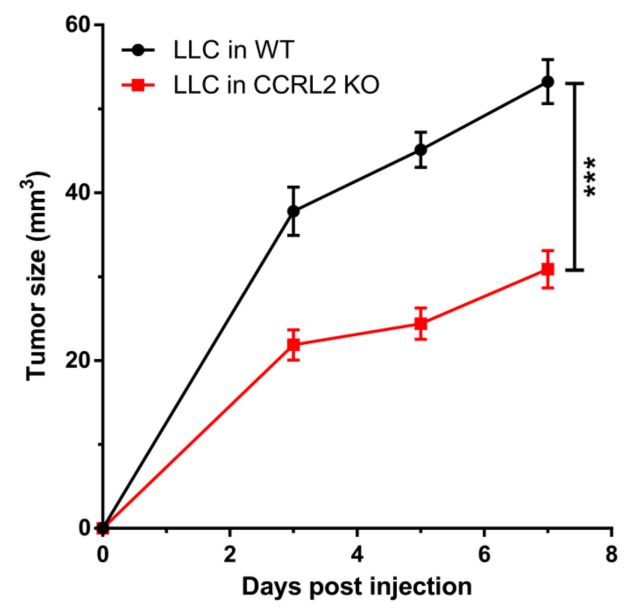

G

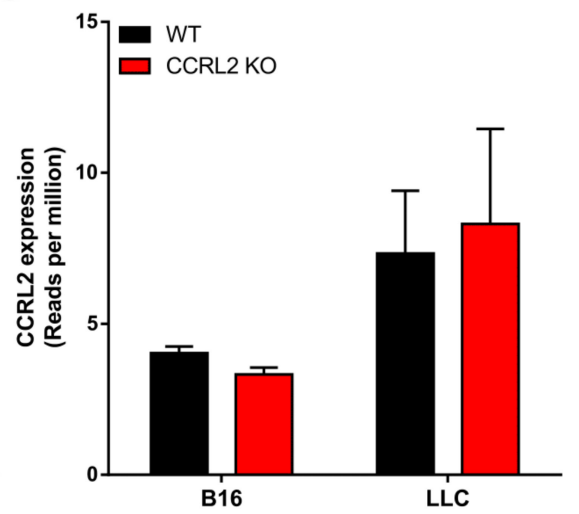

Figure 1. CCRL2 affects tumor growth. (A,B) WT and Ccrl2-deficient mice were subjected to the DMBA/TPA chemical 
carcinogenesis model. The number of tumors per mouse (A) and the proportion of tumors according to size and stage (B) were recorded every other week. (C-F) WT and Ccrl2 KO mice were grafted with B16 melanoma cells (C) and LLC cells (D), and the tumor size was evaluated every other day until day 7. The weight of B16 tumors (E) and LLC tumors (F) was measured following sacrifice of the mice. Data represent the mean \pm SEM, $n \geq 5$ mice per group, ${ }^{* * * *} p<0.0001$, ${ }^{* * *} p<0.001,{ }^{* *} p<0.01$, Mann-Whitney test for all panels. (G) LLC and B16 tumor cells express CCRL2 in vivo. The data representing reads per million were extracted from an RNAseq experiment performed on pools of B16 tumors collected 3 days after the graft and LLC tumors collected 5 days after the graft from WT and Ccrl2 KO mice. For each condition, two pools of 3 tumors were analyzed (mean \pm SEM).

\subsection{The Consequences of Corl2 Loss of Function Are Linked to the Chemerin/CMKLR1 System}

To determine whether CMKLR1 is involved in the effects induced by the knockout of Ccrl2, mice deficient for both Cmklr1 and Ccrl2 (Cmklr1/Ccrl2 KO) and their WT controls were grafted with LLC and B16 cells. No difference in tumor growth was recorded between the two groups (Figure 2A and not shown), suggesting that the effects of CCRL2 on tumor growth in the tumoral graft models are entirely mediated by the chemerin/CMKLR1 system. In the chemical carcinogenesis model, Cmklr1/Ccrl2 KO mice had a phenotype similar to that observed for $\mathrm{Ccrl} 2 \mathrm{KO}$ mice, with tumors appearing in a larger number than in WT mice (Figure 2B,C), although the difference was less important. In this model, it seems that part of the effect of Ccrl2 loss of function is dependent on CMKLR1, but that the phenotype might also be mediated either by the third chemerin receptor GPR1 or by an unidentified chemerin-independent mechanism.

Using a transgenic mouse model overexpressing bioactive chemerin under the control of the keratin $\mathrm{K} 5$ promoter, we have shown recently that expression of chemerin by keratinocytes inhibits tumor development in the DMBA/TPA model, and this effect is mediated by the main receptor of chemerin, CMKLR1 [23]. To determine whether CCRL2 is involved in the protective effects of chemerin, we used mice overexpressing chemerin and deficient for CCRL2 (K5-chemerin/Ccrl2 KO) and subjected them to the DMBA/TPA model. As previously reported, $\mathrm{K} 5$-chemerin mice developed a lower number of tumors than their WT controls, and Ccrl2 knockout increased the number of tumors (Figure 2D). K5-chemerin/Ccrl2 KO mice presented an intermediate phenotype. The number of tumors developed by $\mathrm{K} 5$-chemerin/Ccrl2 $\mathrm{KO}$ mice was relatively similar to that of control mice during the first weeks and stabilized from around week 20 (Figure 2E). These data suggest that CCRL2 contributes to the anti-tumoral effects of chemerin in the overexpression model, and on the other hand, that chemerin overexpression neutralizes the consequences of $\mathrm{Ccrl} 2$ loss of function. 
A

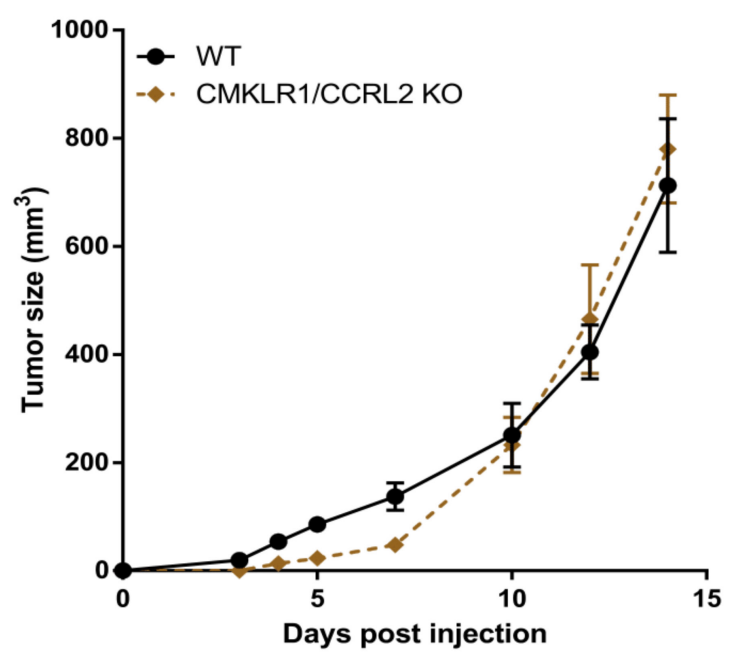

D

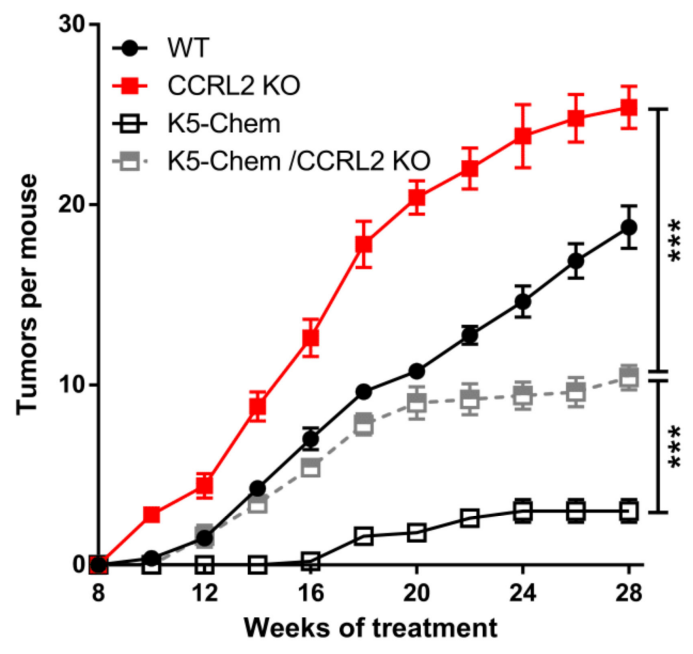

B

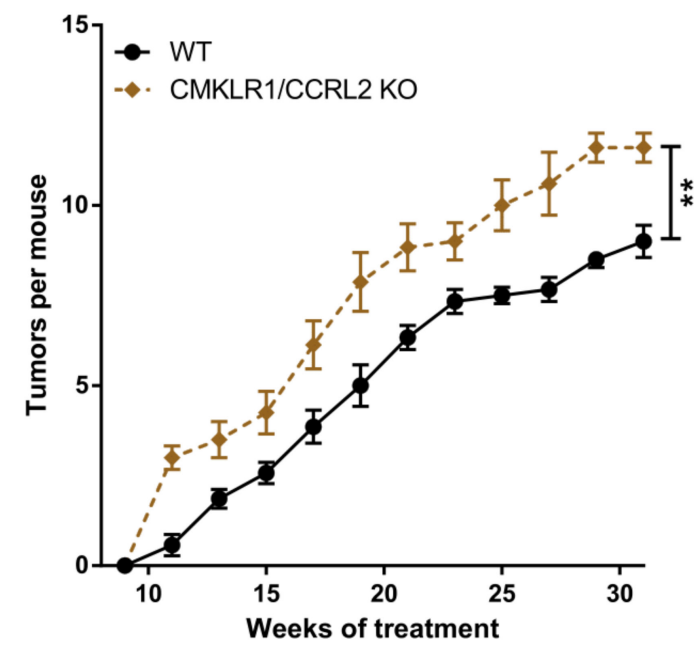

C

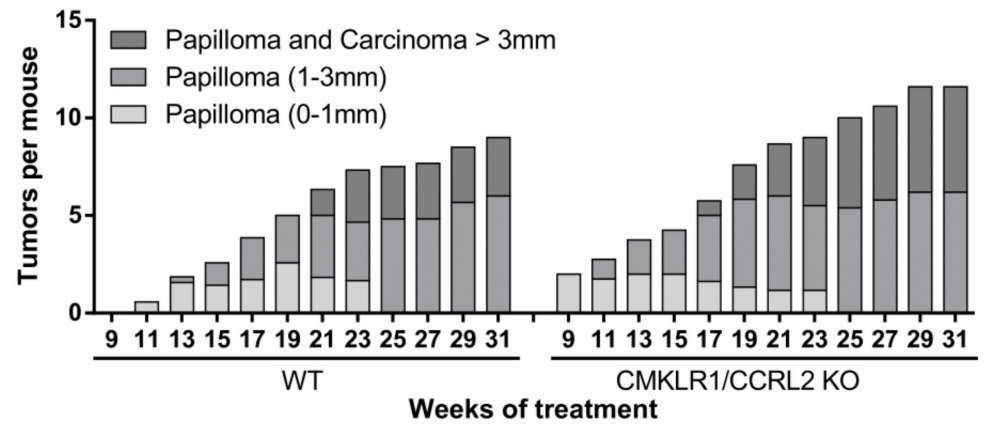

E

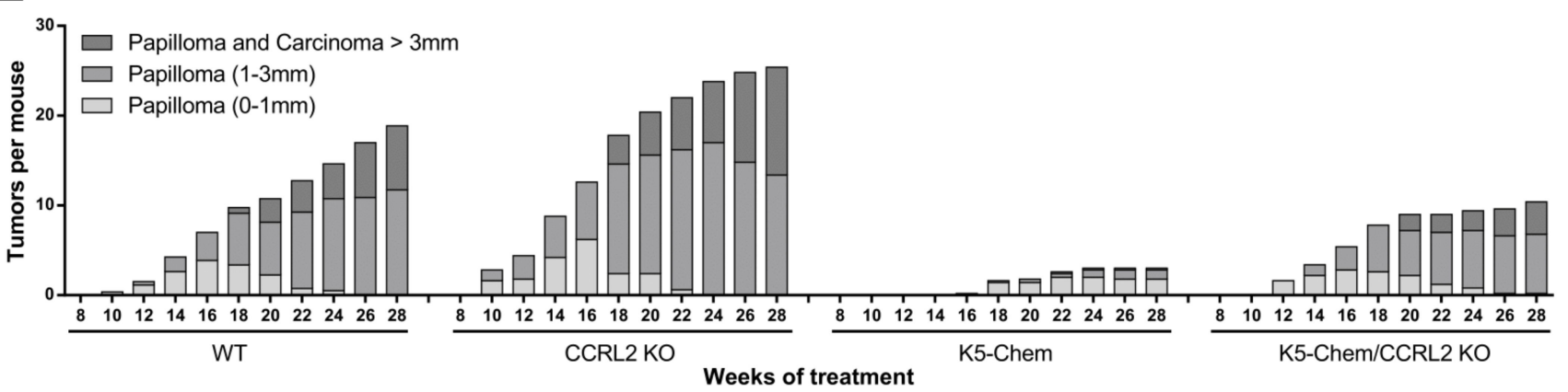

Figure 2. The effects of CCRL2 on tumor growth are mediated by CMKLR1. (A) WT and Cmklr1/Ccrl2 double KO mice were grafted with LLC cells, and the tumor size was measured over time. Representative experiment out of three performed (mean \pm SEM, $n \geq 5$ mice per group). (B,C) WT and Cmklr1/Ccrl2 double KO mice were subjected to the DMBA/TPA chemical carcinogenesis model. The number of tumors per mouse (mean \pm SEM) (B) and the size and the grade of the tumors (C) were recorded. (D,E) WT, K5-chemerin, Ccrl2 KO, and K5-chemerin/Ccrl2 KO mice were subjected to the DMBA/TPA chemical carcinogenesis model. The number of tumors per mouse (mean \pm SEM) (D) and the proportion of tumors according to size and stage (E) were recorded every other week. ${ }^{* *} p<0.001,{ }^{* *} p<0.01$, Mann-Whitney for panels $\mathrm{A}$ and B, one-way ANOVA for panel D. The data are the compilation of three independent experiments with $n \geq 5$ mice per group in each experiment. 


\subsection{Knocking out Ccrl2 in Tumor Cells Restores the Growth of Tumor Grafts in Ccrl2 KO Mice}

Several studies have reported the expression of CCRL2 by tumor cells [17,19], and we demonstrated that B16 and LLC cells express CCRL2 in vivo. Therefore, we investigated the potential effect of CCRL2 expression by tumor cells on tumor growth. We generated LLC and B16 cell lines knocked out for Ccrl2 (B16-Crispr ${ }^{\mathrm{Ccrl} 2}$ and LLC-Crispr ${ }^{\mathrm{C} r l 2}$ cells). The approach combined a mutant Cas9 nickase and a pair of guide RNAs (sgRNA) in order to reduce potential off-target mutagenesis events, which are frequent with wild-type Cas9. The presence of mutations in Ccrl2 alleles was tested in a set of clones by PCR amplification and a DNA heteroduplex mobility assay on polyacrylamide gels, followed by sequencing of the cloned PCR product. Several B16-Crispr ${ }^{C c r l 2}$ and LLC-Crispr ${ }^{C c r l 2}$ cell lines baring frameshift deletions in both alleles were selected (Figure S1). B16-Crispr ${ }^{\mathrm{Ccrl}}$ and LLC-Crispr ${ }^{C c r l 2}$ cells were grafted subcutaneously into the back of WT and Ccrl2 KO mice (Figure 3A-D). B16-Crispr ${ }^{C c r l 2}$ or LLC-Crispr ${ }^{\mathrm{C} c r l 2}$ cells generated tumors of similar size in WT and Ccrl2 $\mathrm{KO}$ mice, while delayed tumor growth was observed in Ccrl2 $\mathrm{KO}$ mice injected with control B16 (Figure 3A,B) or control LLC cells (Figure 3C,D). Similar results were obtained with two independent clones of B16 and LLC cells knocked out for Ccrl2 (data not shown). We evaluated the expression of chemerin and its receptors by qRT-PCR in B16 tumors of Ccrl2 KO and WT mice. CCRL2 expression was moderately affected by the knockout of the host gene, but much more by the knockout of the gene in the tumor cell line (Figure 3E). It was down to background levels when knocked out in both the host and the cell line. There was no significant difference in the expression of chemerin, CMKLR1, and GPR1. These data confirm that most Ccrl2 transcripts in tumors derive from tumor cells and not from the microenvironment, and that this expression by tumor cells is responsible for the delayed tumor growth in Ccrl $2 \mathrm{KO}$ mice.

\subsection{CCRL2 Overexpression by Tumor Cells Delays Tumor Growth}

We next investigated the effect of CCRL2 overexpression by tumor cells on tumor growth in vivo. We generated B16 and LLC clones stably overexpressing a codon-optimized version of mouse Ccrl2. Expression of the gene was confirmed by PCR (data not shown) and FACS analysis. B16-CCRL2 and LLC-CCRL2 lines displayed a strong shift (Figure S2) while CCRL2 was undetectable in control cells and clones knocked out for CCRL2 (B16$\mathrm{Crispr}^{\mathrm{C} c r l 2}$ and LLC-Crispr ${ }^{\mathrm{Ccrl}}$ ) in culture. Mice were injected subcutaneously with control B16 or LLC cells or cell lines overexpressing CCRL2. Overexpression of CCRL2 by tumor cells strongly reduced the growth of B16 and LLC tumors in WT mice (Figure 4 and Figure S3). Similar growth delays were observed with different B16 and LLC clones overexpressing CCRL2 (data not shown). We tested whether these effects were dependent on the presence of functional chemerin, Cmklr1, and Gpr1 genes by using mice knocked out for chemerin, Cmklr1, Gpr1, or Gpr1/Cmklr1. Control B16 and LLC tumors grew similarly in WT, chemerin KO (Figure 4A,B; Figure S3A,B), Cmklr1 KO (Figure 4C,D and Figure S3C,D), Gpr1 KO (Figure S4), and Gpr1/Cmklr1 double KO mice (Figure 4E,F and Figure S3E,F). The consequences of CCRL2 overexpression by tumor cells were partially reversed in chemerin KO, Cmklr1 KO, and Gpr1/Cmklr1 double KO mice (Figure 4 and Figure S3) but unaffected in Gpr1 KO mice (Figure S4). These results suggest that the delayed growth observed for cells overexpressing CCRL2 is partially dependent on the chemerin/CMKLR1 system but independent from GPR1. 
A

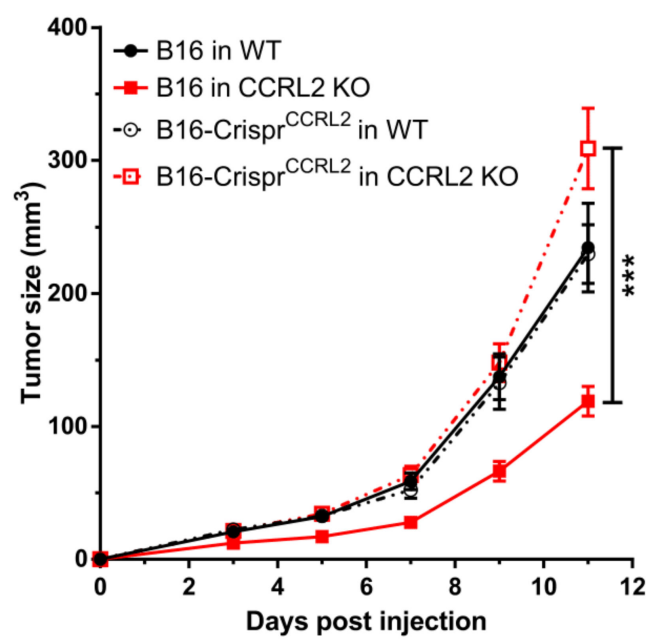

C

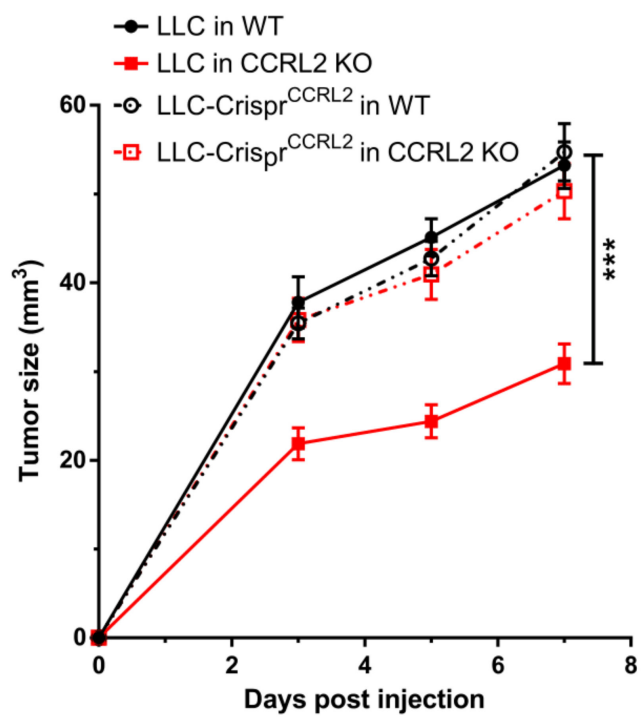

E

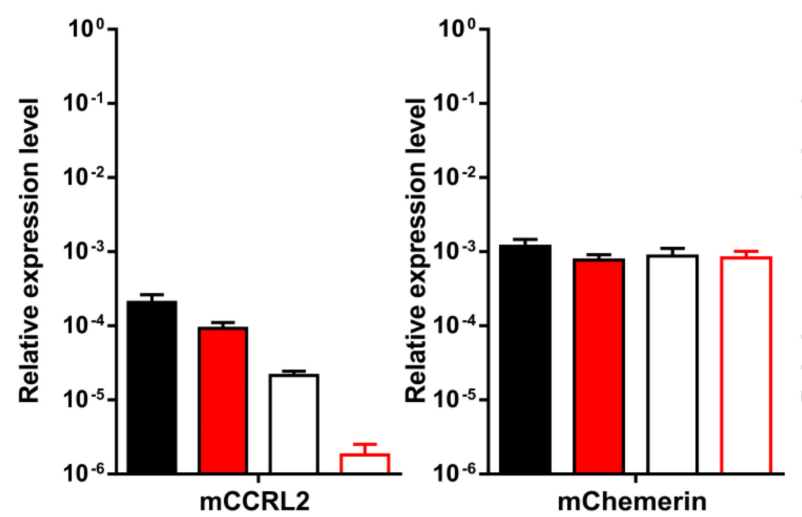

B

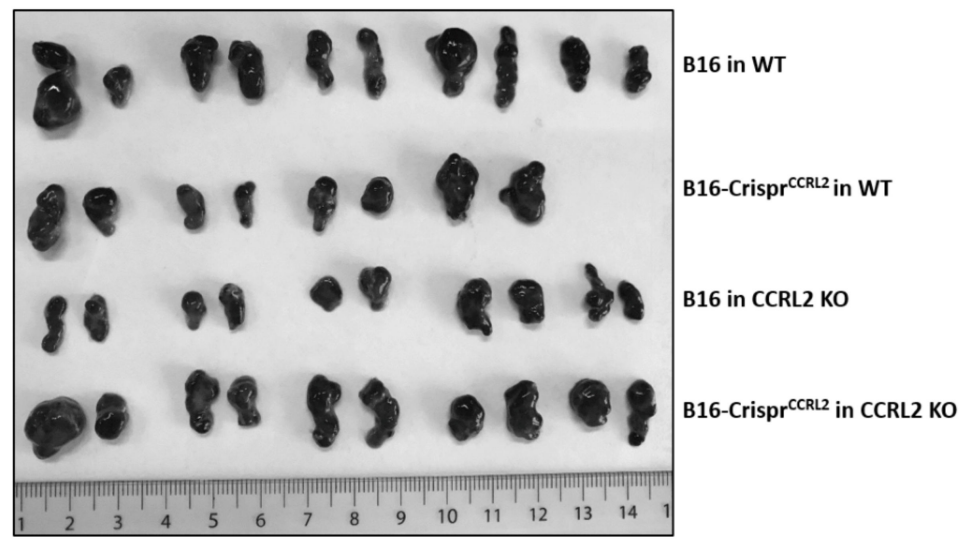

D

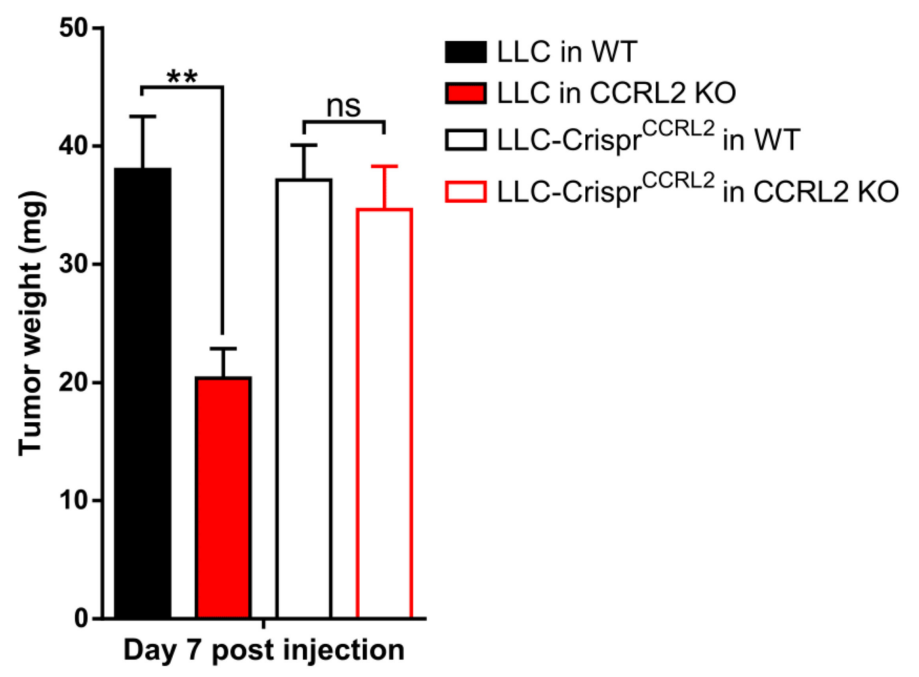

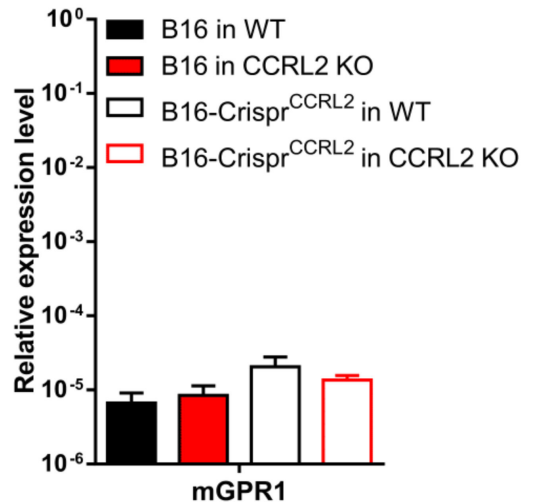

Figure 3. Ccrl2 knockout in tumor cells abolishes the consequences of Ccrl2 loss of function in the host. B16 and LLC clones knocked out for Ccrl2 were generated by the CRISPR/Cas9n technology. (A,B) B16 cells and a B16 clone knocked 
out for Ccrl2 (B16-Crispr ${ }^{C c r l 2}$ ) were grafted to WT and Ccrl2 KO mice. The size of the tumors was monitored up to day 11 (A), and the tumors collected following sacrifice are shown (B). (C,D) LLC cells and an LLC clone knocked out for Ccrl2 (LLC-Crispr ${ }^{C c r l 2}$ ) were grafted to WT and Ccrl2 KO mice. The size of the tumors was monitored up to day 7 (C), and the weight of the tumors measured following sacrifice (D). (E) Chemerin, CMKLR1, CCRL2, and GPR1 transcript levels were evaluated by qRT-PCR in B16 tumors collected on day 11. ${ }^{* *} p<0.001,{ }^{* *} p<0.01$, one-way ANOVA followed by Tukey-Kramer test for all panels. Data from two independent experiments are shown and expressed as mean \pm SEM.

A

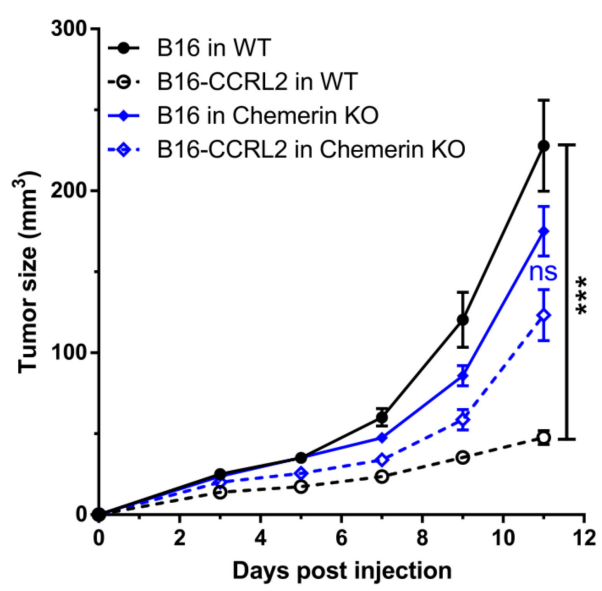

C

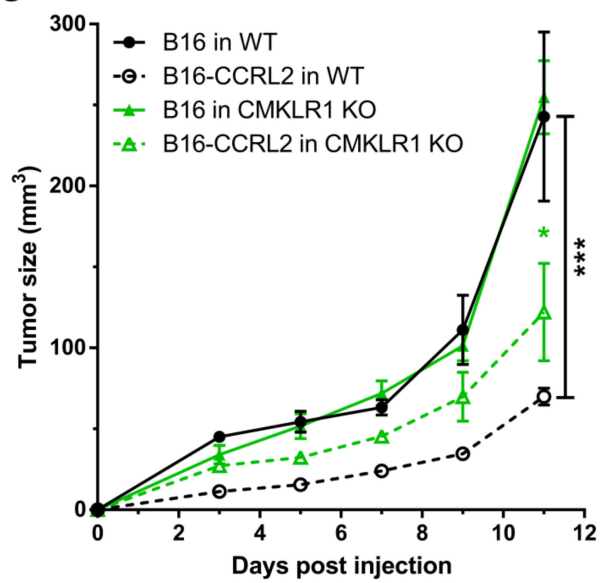

E

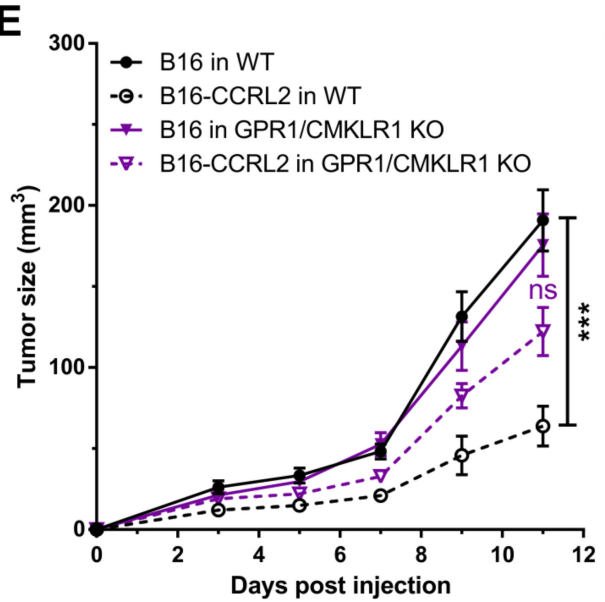

B

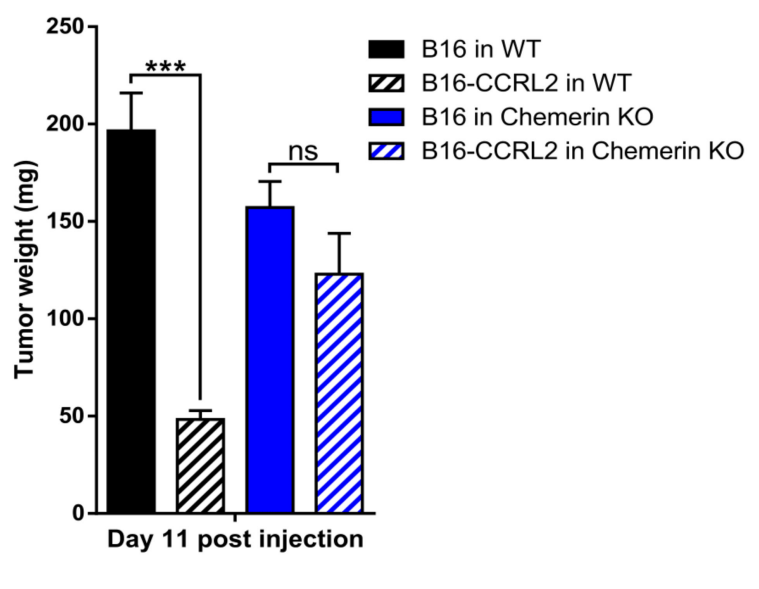

D

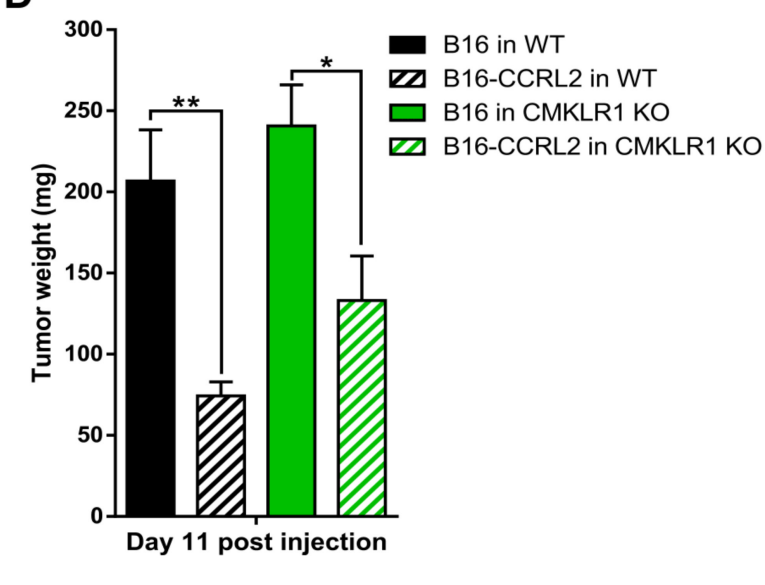

$\mathbf{F}$

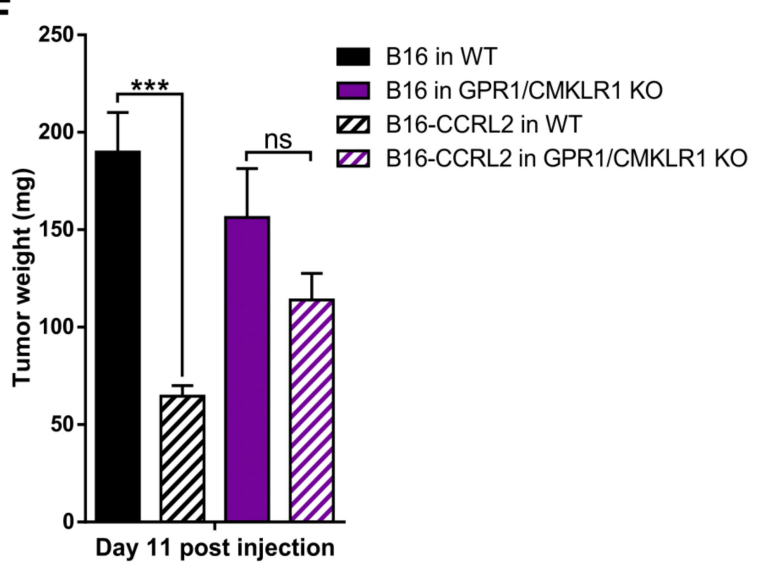

Figure 4. The consequences of CCRL2 overexpression in tumor cells are partially dependent on chemerin and CMKLR1 
but independent of GPR1. WT and chemerin KO (A), Cmklr1 KO (C), and Gpr1/Cmklr1 KO mice (E) were grafted with B16 or B16-CCRL2 cells, and the tumor size measured over time. (B,D,F) The weight of the tumors was measured in each group at day 11 post-graft. The data are the mean \pm SEM of three independent experiments with $\geq 5$ mice per group in each experiment. ${ }^{* * *} p<0.001,{ }^{* *} p<0.01,{ }^{*} p<0.05$, one-way ANOVA followed by Tukey-Kramer test for all panels.

\subsection{Overexpression or Knockout of Ccrl2 Do Not Affect Proliferation of B16 and LLC Cells Ex Vivo}

Receptors such as chemokine receptors can affect tumor cell proliferation via autocrine mechanisms or by influencing the response of other membrane receptors [31,32], while clonal selection following modification of a cell line may also result in differential growth patterns. Therefore, we tested the proliferation properties of the B16 and LLC clones overexpressing CCRL2 or knocked out for the receptor in culture conditions ex vivo. None of the clones tested displayed a detectable change in their cell proliferation rate in culture, as compared to control cells (Figure S5A,B), nor their doubling time (Figure S5C-F). Therefore, CCRL2 expression by tumor cell lines does not modify the proliferation rate of B16 and LLC cells in vitro, and the selection of clones did not result in significant changes in the growth pattern of the cells. Besides, the data displayed in vivo for a single B16 or LLC clone overexpressing CCRL2 or knocked out for the receptor were reproduced for at least one other clone, with no significant difference in the outcome (data not shown).

\subsection{The Recruitment of Immune Cells to Tumors Is Not Affected in Ccrl2 KO Mice}

Various immune cells express CCRL2 [33], including dendritic cells [21] and macrophages [34], and this might affect the trafficking of these cells in response to chemerin $[3,14,35]$. CCRL2 expression by endothelial cells or other cell types may also contribute to the regulation of leukocyte trafficking $[15,20,36]$ while CCRL2 may modify the functional response of other chemoattractant receptors in cells where the two receptors are co-expressed [37]. Therefore, we examined whether Ccrl2 deficiency in the host modifies the recruitment of immune cells to the tumor microenvironment. The proportion of leukocytes was assessed by FACS in tumors collected from WT and Ccrl2 KO mice. No significant changes were observed in the number of $\mathrm{CD}_{4} 5^{+}$cells, nor in the proportion of different leukocyte subpopulations in LLC tumors at day 5 post-graft (Figure 5A). Similar experiments were also performed on B16 tumors and at different time points with similar outcomes (data not shown). Next, we grafted B16, B16-CCRL2, and B16-Crispr ${ }^{\mathrm{C} c r l 2}$ cells to WT and NOD/SCID immunodeficient mice. Tumors overexpressing CCRL2 were significantly smaller than WT B16 and B16Crispr ${ }^{C r l 2}$ tumors in WT mice (Figure 5B-D) but also in NOD/SCID mice (Figure 5C-E). These results support the concept that, in these tumor graft models, CCRL2 acts on tumor growth independently from the recruitment of leucocyte populations. We also assessed, by qRT-PCR, the expression of several mediators of inflammation, including chemokines (CXCL1, CXCL2, CCL2), cytokines (IL-1 $\beta$, IL-6, IL-10, IFN- $\gamma$ ), and proteases (MMP9, MMP10) in B16 and LLC tumors collected from WT and Ccrl2 KO mice. We did not observe any significant differences in the expression of these genes between the two genotypes (data not shown). 
A
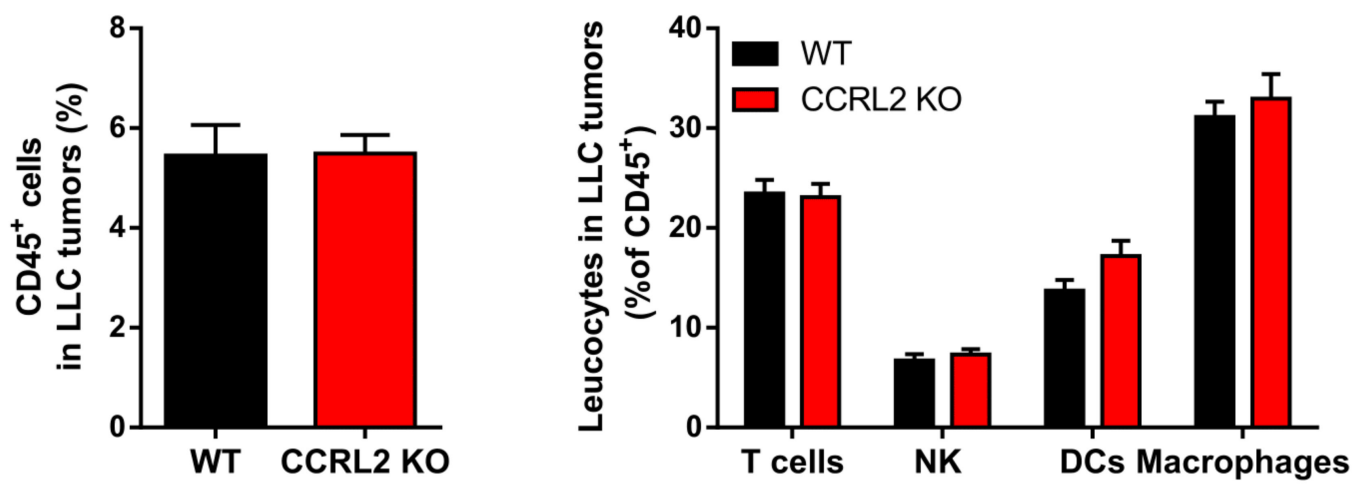

B

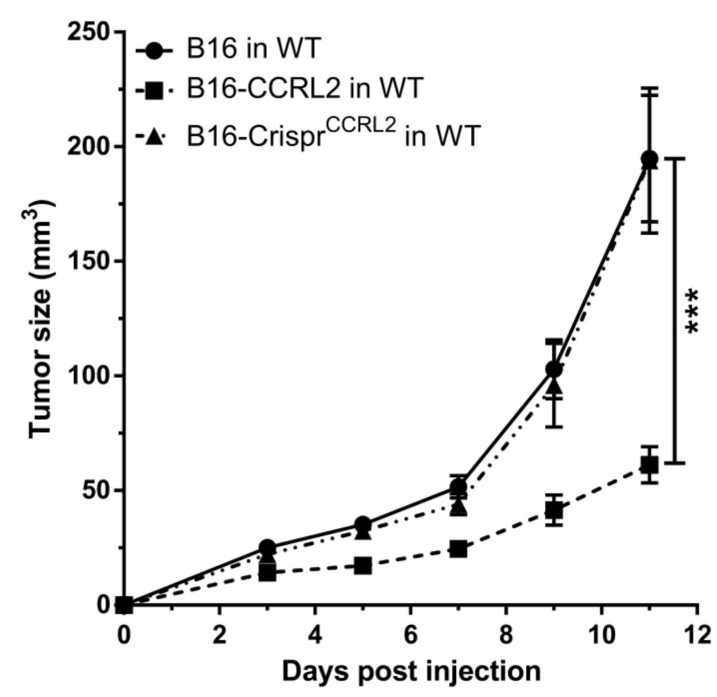

C

D
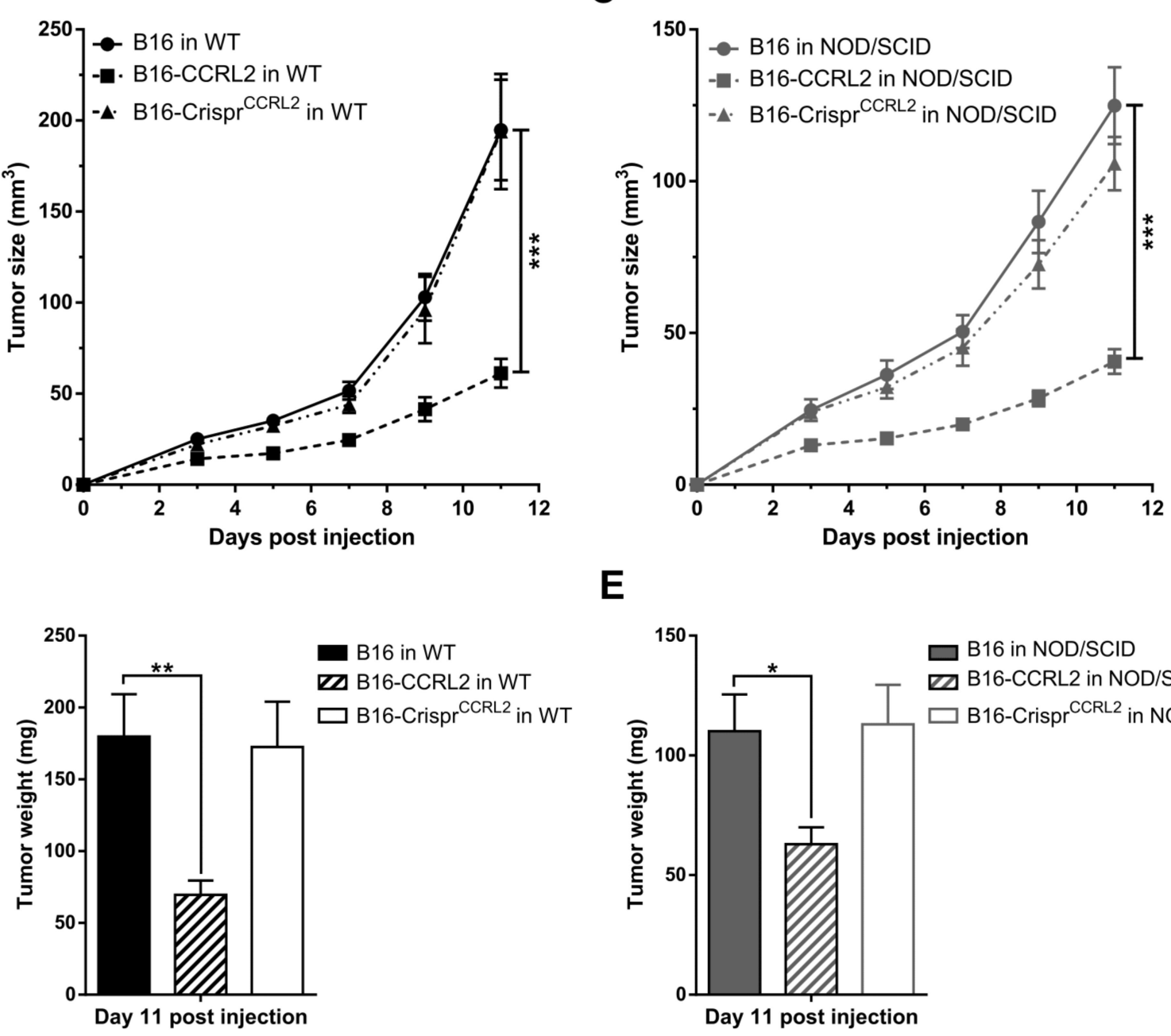

E

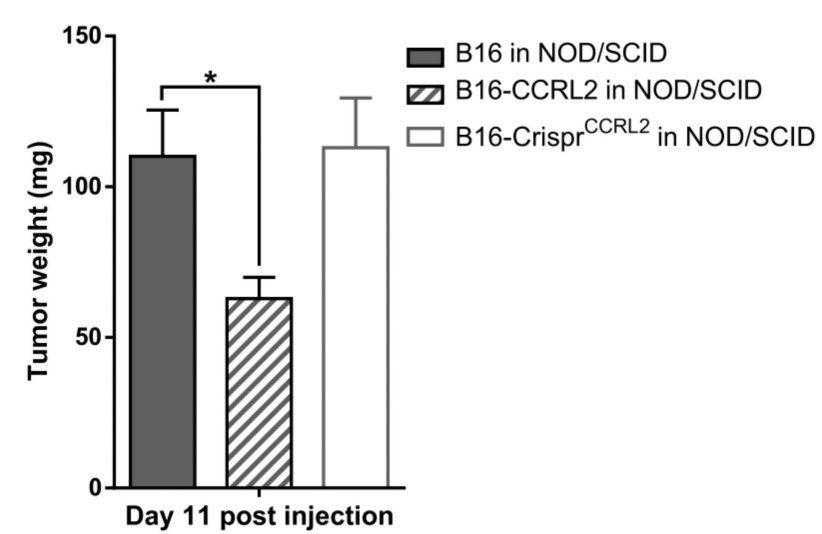

Figure 5. The anti-tumoral effect of CCRL2 is independent of the recruitment of leukocytes. (A) LLC cells were grafted to control and Ccrl $2 \mathrm{KO}$ mice. Five days after the graft, the tumors were collected and the cells analyzed by flow cytometry. The percentage of $\mathrm{CD}_{4} 5^{+}$cells and the proportion of various leukocyte subsets (\% of $\mathrm{CD} 45^{+}$cells) are represented, including T cells $\left(\mathrm{CD}^{+} \mathrm{NK}^{-} 1^{-}\right)$, NK cells $\left(\mathrm{CD} 3^{-} \mathrm{NK} 1.1^{+}\right)$, DCs $\left(\mathrm{CD} 11 \mathrm{c}^{+}\right)$, and macrophages $\left(\mathrm{CD} 11 \mathrm{~b}^{+} \mathrm{CD} 11 \mathrm{c}^{-} \mathrm{Gr}^{-}\right)$. Compilation of three independent experiments with 5 mice per condition in each experiment. (B-E) B16, B16-CCRL2, and B16-CrisprCcrl2 cells were grafted to WT (B) and NOD/SCID mice (C), and the tumor size was monitored daily. The weight of the tumors was measured at day 11 post-injection $(\mathbf{D}, \mathbf{E})$. The results (mean $\pm \mathrm{SEM}$ ) are from three independent experiments with $n$ $=6$ mice per group in each experiment. ${ }^{* *} p<0.001,{ }^{* *} p<0.01,{ }^{*} p<0.05$, Mann-Whitney for panels A and B, one-way ANOVA followed by Tukey-Kramer test for all other panels. 


\subsection{CCRL2-Expressing Cells Concentrate Chemerin and Activate CMKLR1 ${ }^{+}$Cells}

CCRL2 binds chemerin through its $\mathrm{N}$-terminal domain, leaving the carboxy-terminal peptide critical for cell signaling accessible for an interaction with cells expressing CMKLR1 [14]. CCRL2 acts, therefore, as a chemerin-presenting molecule on barrier cells [36,38]. We hypothesized that CCRL2 (over)expressed by tumor cells might increase the local concentration of chemerin in the tumor and enhance the functional response of CMKLR1expressing cells in the microenvironment. To test this hypothesis, we compared the ability of WT B16 cells, or clones overexpressing CCRL2 or knocked out for Ccrl2, to stimulate CHO-K1 cells expressing CMKLR1 following a pre-incubation with bioactive chemerin. Only cells overexpressing CCRL2 and loaded with chemerin were able to trigger calcium mobilization in CMKLR1-expressing CHO-K1 cells, as measured in an aequorin-based calcium mobilization assay (Figure 6A). WT B16 and B16-Crispr ${ }^{\mathrm{C} c r l 2}$ cells did not generate a specific response in this assay. We next tested whether tumor cells overexpressing CCRL2 could concentrate chemerin on their surface. The various B16 clones, as well as CHO-K1 cells expressing CMKLR1 used as a positive control, were incubated for $2 \mathrm{~h}$ at $37^{\circ} \mathrm{C}$ in the presence of $10 \mathrm{nM}$ of a GFP-chemerin fusion protein. CMKLR1 $1^{+} \mathrm{CHO}-\mathrm{K} 1$ cells bound and internalized GFP-chemerin very efficiently (data not shown), as previously reported [39]. An anti-GFP antibody allowed us to demonstrate efficient binding of GFP-chemerin onto B16-CCRL2 cells (Figure 6B), but no significant internalization of the ligand was observed. In contrast, WT B16 and B16-CrisprCcrl2 cells ex vivo did not bind detectable levels of GFP-chemerin in this assay (Figure 6B).

\subsection{CCRL2 Expression by Tumor Cells Impairs Tumor Vascularization}

Neovascularization is an important component of tumor progression, as it provides oxygen and nutrients to the growing tumor. CCRL2 and CMKLR1 are expressed by endothelial cells, and we demonstrated previously that chemerin expression by tumor cells inhibits tumor angiogenesis, thereby promoting cell death and a growth delay [10]. We therefore investigated the histology of tumors collected from WT and Ccrl2 KO mice. B16 or LLC tumors grown in Ccrl2 $\mathrm{KO}$ mice presented larger necrotic areas compared to tumors from control mice (Figure 7A,B). Additionally, the relative surface of $\mathrm{CD} 31^{+}$staining was significantly smaller in tumors from Ccrl2 KO mice compared to WT mice (Figure 7B). The knockout of $\mathrm{Ccrl} 2$ in tumor cells restored the vascularization and necrotic area in $\mathrm{Ccrl} 2 \mathrm{KO}$ mice to a level similar to that observed in WT mice (Figure 7A,B). We next tested whether tumor angiogenesis was also affected by the overexpression of CCRL2 in tumor cells. Tumors overexpressing CCRL2 collected from WT mice showed a significant reduction of the $\mathrm{CD} 31^{+}$area and larger necrotic regions compared to control tumors (Figure 7C-F and Figure S6A-D). The consequences of CCRL2 overexpression on neoangiogenesis were mostly reversed in tumors collected from chemerin $\mathrm{KO}, \mathrm{Cmklr1} \mathrm{KO}$, and Grr1/Cmklr1 KO mice, in which no significant differences in the $\mathrm{CD} 31^{+}$and necrosis areas were seen as compared to WT tumors (Figure 7C-F and Figure S6A-D). 
A

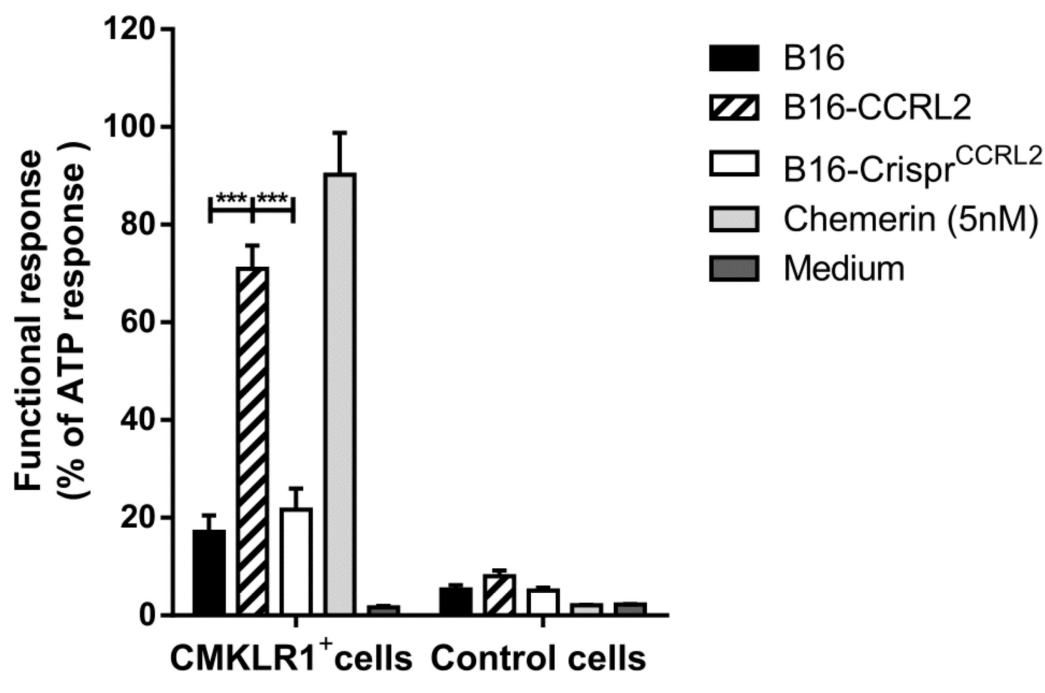

B
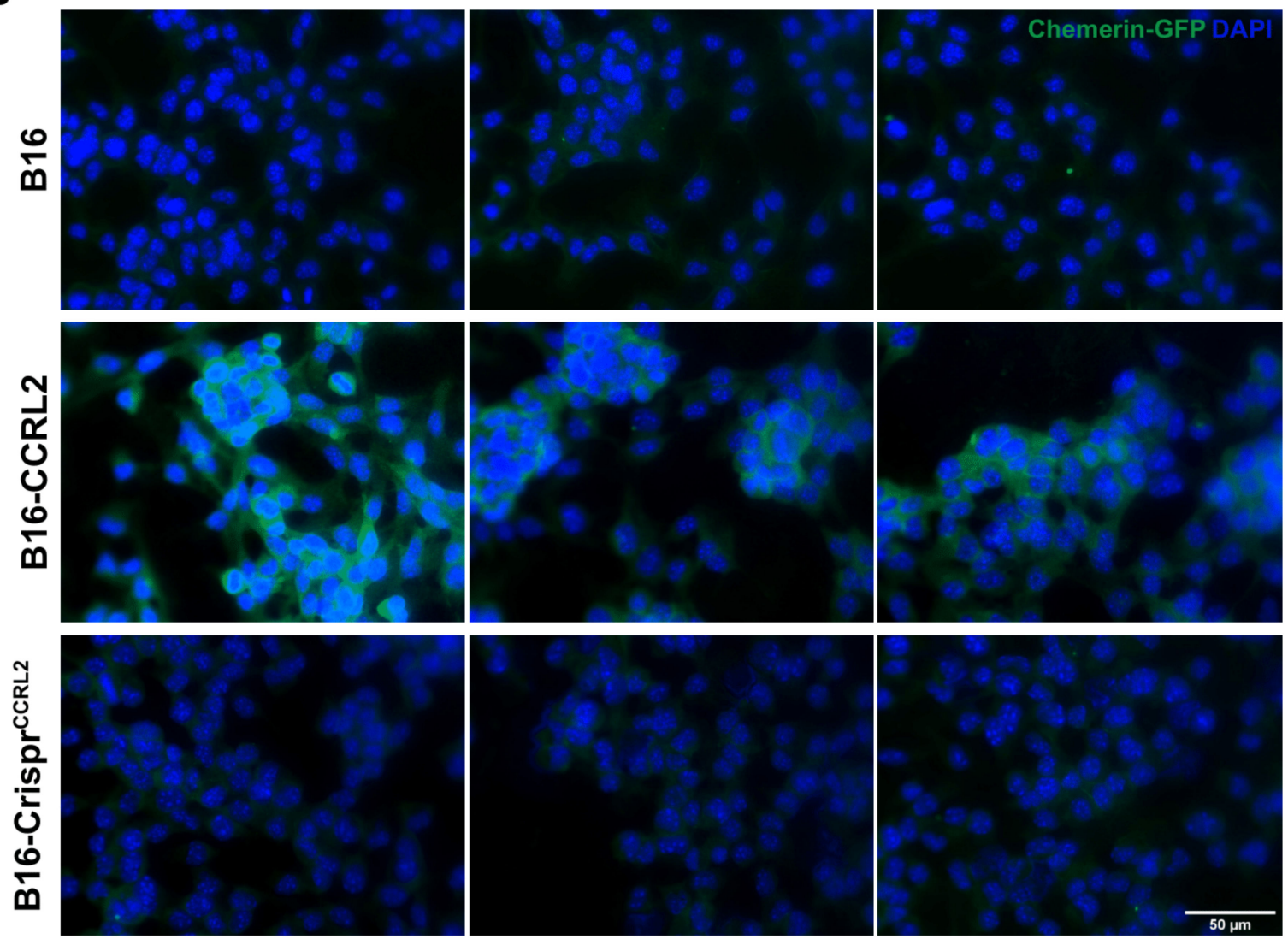

Figure 6. CCRL2 expressed by tumor cells binds and presents chemerin to other cells. (A) B16, B16-CCRL2, and B16Crispr ${ }^{C r l 2}$ cells were preincubated with chemerin, washed and then mixed with CHO-K1 cells expressing or not CMKLR1. The functional response of the cells in the aequorin-based calcium mobilization assay was recorded as a luminescent signal and normalized to the response obtained for $10 \mu \mathrm{M}$ ATP. The data (mean $\pm \mathrm{SEM}$ ) represent the pool of three independent experiments with 3 wells per condition in each experiment. ${ }^{* * *} p<0.001$, one-way ANOVA followed by Tukey-Kramer test. (B) B16, B16-CCRL2, and B16-CrisprCcrl2 cells were incubated for $2 \mathrm{~h}$ at $37^{\circ} \mathrm{C}$ with $10 \mathrm{nM} \mathrm{GFP-chemerin.} \mathrm{The} \mathrm{cells}$ were washed to remove unbound chemerin, fixed, incubated overnight at $4{ }^{\circ} \mathrm{C}$ with an anti-mouse GFP, then $2 \mathrm{~h}$ at room temperature with a Cy3-conjugated goat anti-rabbit IgG. The presence of GFP-chemerin was monitored by fluorescent microscopy. The data are representative of 3 individual experiments with similar results. Scale bars $=50 \mu \mathrm{m}$. 
A

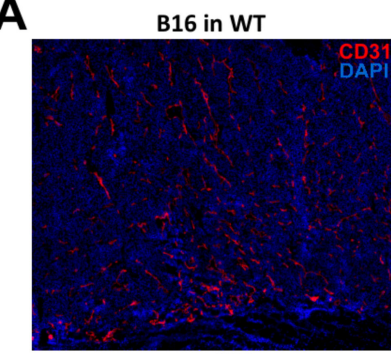

B16-Crispr ${ }^{\text {CCRL2 }}$ in WT

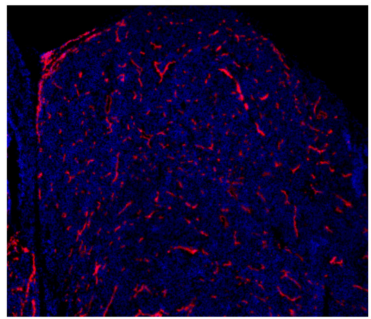

B

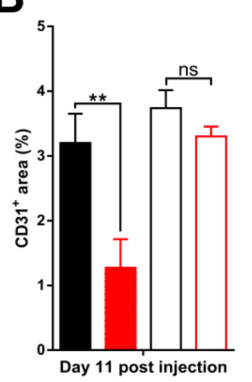

E
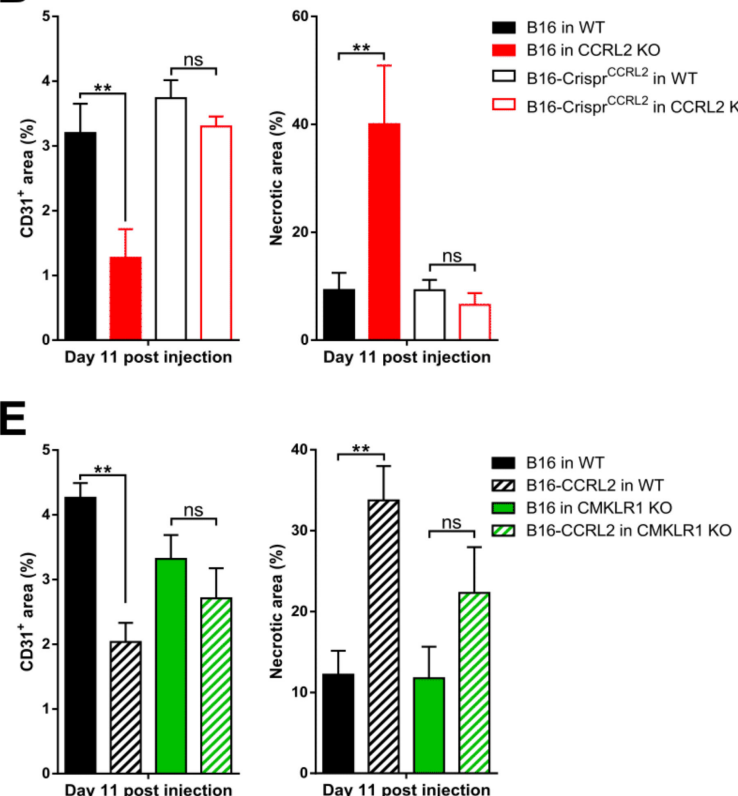

G

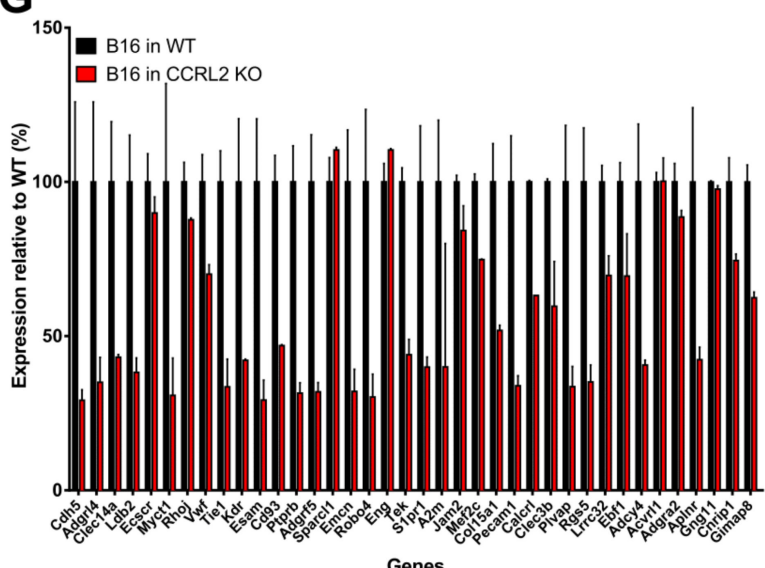

C

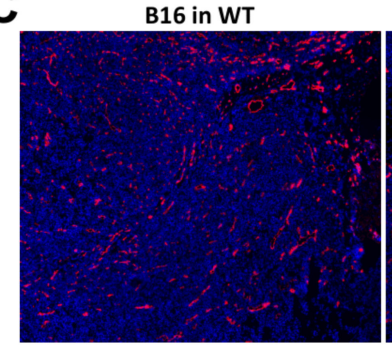

B16-CCRL2 in WT

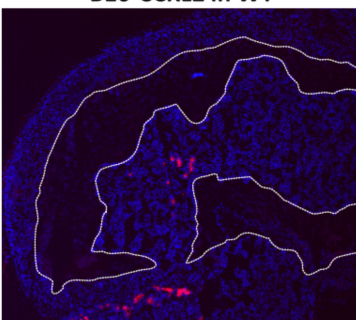

D
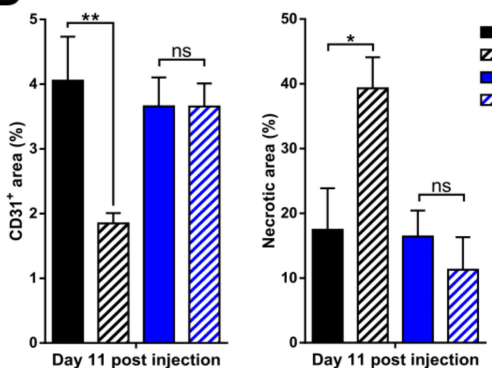

$\mathbf{F}$
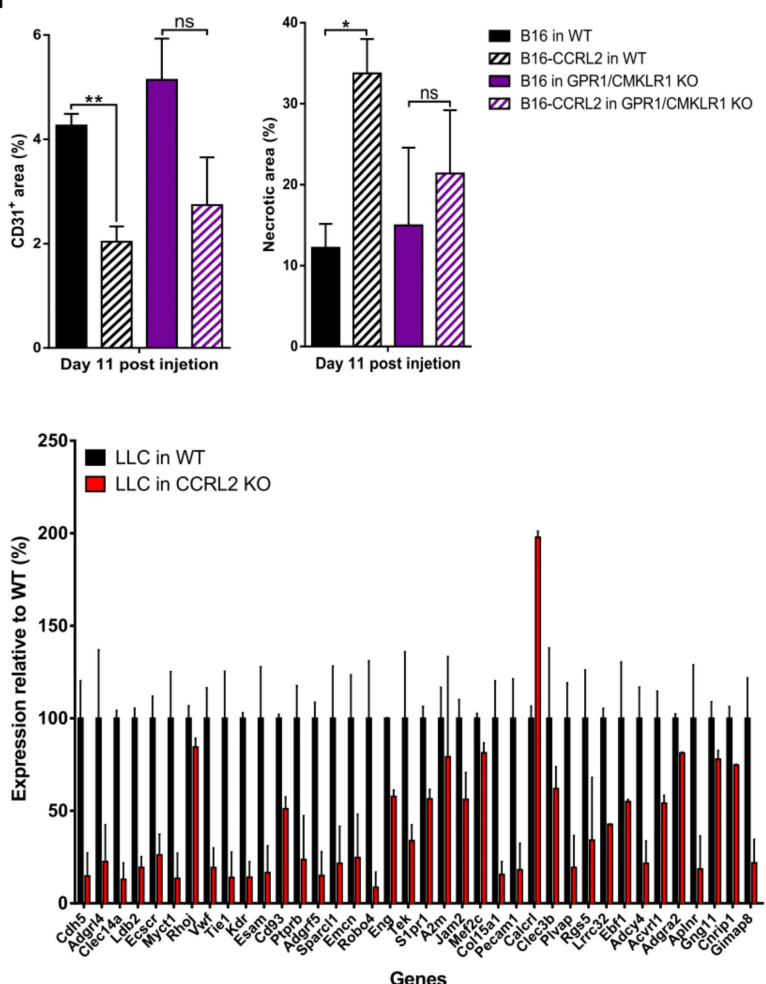

Figure 7. CCRL2 expression by tumor cells regulates neoangiogenesis. (A) Immunostaining of CD31 in B16 and B16- 
Crispr ${ }^{C c r l 2}$ tumors from WT and Ccrl2 $\mathrm{KO}$ mice collected at day 11 post-injection. (B) Relative $\mathrm{CD}^{+} 1^{+}$and necrosis area in B16 and B16-Crispr ${ }^{C c r l 2}$ tumors collected from WT and Ccrl2 KO mice at day 11. (C) Immunostaining of CD31 in B16 and B16-CCRL2 tumors from WT and chemerin KO mice collected at day 11 post-injection. (D) Relative CD31 ${ }^{+}$and necrosis area in B16 and B16-CCRL2 tumors collected from WT and chemerin KO mice at day 11. (E) Relative CD31 ${ }^{+}$and necrosis area in B16 and B16-CCRL2 tumors collected from WT and Cmklr1 KO mice at day 11. (F) Relative CD31 ${ }^{+}$and necrosis area in B16 and B16-CCRL2 tumors collected from WT and Gpr1/Cmklr1 KO mice at day 11. The data (mean \pm SEM) represent the pool of three independent experiments with $\geq 5$ mice per group in each experiment. ${ }^{* *} p<0.01,{ }^{*} p<0.05$, one-way ANOVA followed by Tukey-Kramer test for panels B-F. Scale bars $=500 \mu \mathrm{m}$. (G) Relative expression of the first 38 genes of an angiogenesis signature (described for human cancer types, Masiero et al. 2013) in B16 (left panel) and LLC tumors (right panel) collected from WT and Ccrl2 KO mice. These data were extracted from RNAseq experiments. For each condition, two pools of 3 tumors, collected respectively at days 3 and 5 for B16 and LLC tumors, were analyzed. The data (mean \pm SEM) were normalized relative to the gene expression in WT mice $(100 \%)$.

We performed RNAseq analyses on B16 and LLC tumors from Ccrl2 KO and WT mice, isolated at day 5 post-graft. We observed that B16 and LLC tumors from Ccrl2deficient mice displayed a significant downregulation of an angiogenesis signature of 38 genes (Figure 7G) described for a set of human cancer types [40]. However, there were no significant changes in the expression of key pro-angiogenic factors involved in tumoral angiogenesis such as VEGF-A or FGF-2 (data not shown). Altogether, these results show that exclusive or higher CCRL2 expression in tumor cells relative to the host concentrates chemerin locally and inhibits tumor neoangiogenesis, with consequences similar to those resulting from the expression of bioactive chemerin by tumor cells or by the host.

\section{Discussion}

CCRL2 was shown to regulate inflammatory reactions in diverse pathological conditions, including hypersensitivity reactions, arthritis, and experimental autoimmune encephalitis [21,36,37,41], but its role in cancer has been investigated in few studies so far. Increased CCRL2 expression was described in glioma tumors and glioma cell lines [17]. CCRL2 overexpression did not modify the proliferation of the human glioblastoma cell lines U87MG and U373MG in vitro but increased the migration and invasive properties of the cells. The molecular mechanisms and signaling pathways involved were however not determined. High levels of CCRL2 and CCR1 were also found in liver metastases of colorectal carcinoma in rats, but the consequences of CCRL2 expression appeared limited in human primary colorectal carcinoma cells in terms of proliferation, clonogenic capacity, migration, and survival [19]. High expression of CCRL2 was also described in prostate tumors and the PC-3 prostate cancer cell lines [42]. Highly invasive human breast cancer cell lines (MDA-MB-231 and BT-549) were shown to express low levels of CCRL2, and overexpression of the receptor negatively affected the growth of these cells in vitro and in vivo, as well as their chemotactic response to CCL2 and invasive properties [18]. In addition, CCRL2 expression decreased the phosphorylation of p38 MAPK triggered by CCL2 and restored E-cadherin expression. These observations suggest that CCRL2 may act as a tumor suppressor in human breast cancer cells, while displaying pro-tumoral activities in other cancer types. A recent study supported further the antitumoral role of CCRL2 in urethane-induced and mutant Kras models of lung tumor [20]. The delayed tumor progression observed in $\mathrm{Ccrl} 2 \mathrm{KO}$ mice was associated with a reduction of the recruitment of NK cells, and the involvement of endothelial cells in this phenotype was proposed [20].

In the present study, we analyzed the consequences of Ccrl2 loss of function in different mouse models of cancer, namely the DMBA/TPA chemical model of skin carcinogenesis and the B16 and LLC graft models. The knockout of Ccrl2 in mice accelerated the development of tumors in the two-stage skin carcinogenesis model. In contrast, $\mathrm{Ccrl2}$ deficiency reduced the growth of B16 and LLC tumors in graft models. One significant difference between these models is that $\mathrm{Ccrl} 2$ is not knocked out in the tumor cells in the graft models, while it is absent from all cell types in the chemical carcinogenesis paradigm. Although B16 and LLC do not express high levels of CCRL2 in culture conditions ex vivo, we could 
demonstrate by qRT-PCR and RNAseq experiments that tumors generated by both cell lines in vivo express significant levels of the receptor, with no significant differences between Ccrl2 KO and WT mice. This demonstrated unambiguously that the expression of CCRL2 in tumors could be attributed mostly to tumor cells and not to the microenvironment. LLC and B16 cells therefore express CCRL2 in vivo, and this expression is likely due to the inflammatory context in the tumor microenvironment, since CCRL2 expression is well known to be strongly upregulated by inflammatory signals in various cell types. This observation opened the possibility that the growth delay observed for grafts in Ccrl2-deficient mice might be linked to the exclusive expression of CCRL2 by the tumor cells, resulting in a local concentration of the available bioactive chemerin and enabling chemerin to display its anti-tumoral properties through CMKLR1-expressing cells.

To test this hypothesis, we generated B16 and LLC cell lines in which both Ccrl2 alleles were inactivated by early frame shifts. Knockout of the $\mathrm{C} c \mathrm{rl} 2$ gene in the tumoral cell lines did not modify the growth of tumors in wild-type mice but abrogated the growth delay that was observed in Ccrl2 KO mice grafted with unmodified B16 and LLC cells. These observations validated the proposed model, supporting the role of differential CCRL2 expression across different parts of the body for directing the biological effects of chemerin to specific environments. The consequences of $\mathrm{C} c r l 2$ inactivation in the host were also abrogated by the simultaneous knockout of Cmklr1, demonstrating that the phenotype is mediated through the chemerin/CMKLR1 system.

We further investigated the role of CCRL2 on the activity of the chemerin-CMKLR1 axis in tumor progression by testing tumoral cell lines overexpressing CCRL2. Overexpression of CCRL2 by B16 and LLC cells resulted in a slower growth of the tumors in vivo, without affecting the proliferation of cells in culture ex vivo. This effect was largely reversed by the knockout of the chemerin or Cmklr1 genes in the host, demonstrating the role of the chemerin/CMKLR1 system, while knockout of Gpr1 did not alter the phenotype. Part of the effects of CCRL2 overexpression remained, however, in Cmklr1 and chemerin KO mice, suggesting the contribution of a second mechanism that is presently unidentified. This effect might be linked to changes in the functional response of the cells to other membrane receptors, as previously described for atypical chemokine receptors [11].

In order to validate further the model of chemerin presentation by CCRL2, we could demonstrate the binding of a GFP-chemerin fusion protein on the surface of CCRL2overexpressing tumor cells by immunofluorescence. We also demonstrated that tumor cells overexpressing CCRL2 can display chemerin on their surface in a stable manner, allowing them to trigger functional responses (calcium mobilization in this case) from cells expressing CMKLR1. The results support further that the anti-tumoral effects of CCRL2, when expressed by tumor cells, are mediated by the local concentration of chemerin in the tumor. Such a mechanism was previously described by Monnier et al., showing that CCRL2, expressed by endothelial cells in inflammatory conditions, captured and concentrated chemerin on their surface, contributing thereby to the recruitment of leucocyte populations expressing CMKLR1 and regulating the concentration of the chemoattractant molecule in plasma [15].

We demonstrated previously that the expression of a bioactive form of chemerin by B16 melanoma and Lewis lung carcinoma (LLC) cells delays tumor growth in vivo [10]. A similar tumor growth delay was observed when bioactive chemerin is expressed in the basal keratinocytes of the host mice. These effects did not involve the recruitment of leukocyte populations to the tumors. It was rather found that chemerin prevents efficient angiogenesis in growing tumors, resulting in hypoxia and an increase in necrotic cell death. The antitumoral effect of chemerin was entirely mediated through CMKLR1. The phenotype of the tumors overexpressing chemerin and of the tumors overexpressing CCRL2 or grown in Ccrl2-deficient mice, as described in the present work, were very similar. We did not observe in any of these situations a significant change in the leucocyte populations recruited to the tumors, and similar growth delays were seen in immunodeficient mice. In addition, in all these settings, we observed a significant decrease of the vascularization of the tumors, 
and an increase of necrotic and hypoxic areas in these tumors. RNAseq experiments run on B16 and LLC tumors grown in Ccrl2 $\mathrm{KO}$ mice confirmed a 50\% reduction of an angiogenesis signature, an observation similar to that made previously on chemerinexpressing tumors [10]. The anti-angiogenic effects of chemerin through a direct effect on endothelial cells has been analyzed elsewhere in vitro and in vivo. We showed that chemerin can promote the regression of neovessels during the development of the vascular retinal network, reduce the efficiency of reperfusion in the hind limb ischemia model [43], and inhibit the angiogenesis process of human umbilical vein endothelial cells (HUVEC) in the bead sprouting assay [10]. These data contrast with previous reports in the literature claiming a pro-angiogenic effect of chemerin [44-46], observations that we could not reproduce.

All our data support therefore a model in which a higher expression of CCRL2 in tumor cells affects dramatically the distribution of bioactive chemerin, resulting in its concentration in the area (the tumor) in which CCRL2 expression is the highest. As a result, the cells expressing CMKLR1 are locally stimulated, resulting in a phenotype similar to that observed when chemerin is overexpressed in the tumor. Cell types reported to express CMKLR1 and contributing to the tumor micro-environment include macrophages, myeloid and plasmacytoid dendritic cells, NK cells, endothelial cells, smooth muscle cells, and adipocytes [3,4]. In our tumor graft models, this chemerin bioactivity is essentially targeted toward endothelial cells, resulting in an impairment of the tumor vascularization, leading to hypoxia and cell death by apoptosis and necrosis. A similar mechanism might affect the recruitment or activation of other cells expressing CMKLR1, such as NK cells, although we did not detect such a change in our present models.

In the DMBA/TPA model, we cannot exclude a contribution of leucocyte populations, but the higher number of tumors observed in Ccrl2 $\mathrm{KO}$ mice might be linked also to changes in the distribution of chemerin in the tumor microenvironment. Indeed, the DMBA/TPA model is based on the mutagenic properties of DMBA but requires also the pro-inflammatory and proliferative properties of TPA, as a result of protein kinase $C$ activation. The chronic local skin inflammatory process is expected to generate bioactive chemerin from its precursor, as a result of neutrophil recruitment [47], and to upregulate CCRL2 in many cell populations. It is therefore likely that active chemerin is retained locally by CCRL2 and can exert its anti-tumoral effect as the result of this local concentration. However, in the absence of CCRL2, active chemerin may leak out of the inflamed tissue much more easily, thereby explaining the faster tumor progression in $\mathrm{Ccrl} 2 \mathrm{KO}$ mice.

\section{Conclusions}

The present work supports the important role of CCRL2 in tuning the activity of the chemerin/CMKLR1 system. The modulation of angiogenesis observed in our tumor grafts complements other effects of CCRL2 on the tumor microenvironment observed in other models. The properties and roles of CCRL2 are very similar to those attributed to atypical chemokine receptors, modulating the anatomical distribution of their respective ligands and thereby modifying the activity of the cognate functional receptors. In this context, CCRL2 appears as a new player in tumorigenesis and angiogenesis, and its expression should be taken into account in the development of potential therapeutic strategies targeting the chemerin/CMKLR1 system.

Supplementary Materials: The following are available online at https:/ / www.mdpi.com/article/10 .3390 / cancers13195000/s1. Figure S1: Knockout of the Ccrl2 gene in B16 and LLC cells. Figure S2: CCRL2 expression in B16 and LLC tumor cell lines. Figure S3: The consequences of CCRL2 overexpression in LLC tumors are partially dependent on chemerin and CMKLR1 but independent of GPR1. Figure S4: The anti-tumoral effect of CCRL2 is independent of GPR1. Figure S5: Overexpression or knockout of Ccrl2 do not affect the proliferation rate of B16 and LLC clones in vitro. Figure S6: CCRL2 expression by LLC cells regulates neoangiogenesis.

Author Contributions: Conceptualization, V.W., M.P.; methodology, D.A.D., V.R., I.D.-V.; validation, D.A.D., M.P.; formal analysis, D.A.D., V.R., I.D.-V., F.L.; investigation, D.A.D., V.R., I.D.-V., M.V., A.R., A.L., 
F.L.; resources, A.D.P., S.S.; writing—original draft preparation, D.A.D.; writing—review and editing, V.W., S.S., M.P.; visualization, D.A.D., M.P.; supervision, M.P.; project administration, M.P.; funding acquisition, S.S., M.P. All authors have read and agreed to the published version of the manuscript.

Funding: This research was funded by the Fonds de la Recherche Scientifique Médicale of Belgium to M.P. This work was also partially supported by AIRC (Associazione Italiana Ricerca sul Cancro) under IG 2017 (ID. 20776 project) and by MIUR (Ministero per l'Università e Ricerca) Italy to S.S., D.A.D. and I.D.V. were supported by FNRS-Télévie grants and the foundations of Rose and Jean Hoguet and David and Alice Van Buuren.

Institutional Review Board Statement: The study was conducted according to the European guidelines and local regulations and approved by the ethics committee (Commission d'Ethique du Bien-Etre Animal, CEBEA) of the ULB Medical School (protocol 660N approved on 1 March 2018).

Informed Consent Statement: Not applicable.

Data Availability Statement: The data of RNA sequencing have been deposited in NCBI's Gene Expression Omnibus and are accessible through GEO Series accession number GSE183914.

Acknowledgments: The authors thank Mustapha Chaouni for expert technical assistance.

Conflicts of Interest: M.P. is CEO of the Biotech Company Gepeceron, a spin-off of the Université Libre de Bruxelles. All other authors declare no conflict of interests. The funders had no role in the design of the study, in the collection, analyses, or interpretation of data, in the writing of the manuscript, or in the decision to publish the results.

\section{References}

1. Hanahan, D.; Weinberg, R.A. Hallmarks of Cancer: The Next Generation. Cell 2011, 144, 646-674. [CrossRef]

2. Mantovani, A. The chemokine system: Redundancy for robust outputs. Immunol. Today 1999, 20, 254-257. [CrossRef]

3. Bondue, B.; Wittamer, V.; Parmentier, M. Chemerin and its receptors in leukocyte trafficking, inflammation and metabolism. Cytokine Growth Factor Rev. 2011, 22, 331-338. [CrossRef] [PubMed]

4. Kennedy, A.; Davenport, A.P. International Union of Basic and Clinical Pharmacology CIII: Chemerin Receptors CMKLR1 (Chemerin1) and GPR1 (Chemerin2) Nomenclature, Pharmacology, and Function. Pharmacol. Rev. 2017, 70, 174-196. [CrossRef]

5. Goralski, K.B.; Jackson, A.E.; McKeown, B.T.; Sinal, C.J. More Than an Adipokine: The Complex Roles of Chemerin Signaling in Cancer. Int. J. Mol. Sci. 2019, 20, 4778. [CrossRef]

6. Zheng, Y.; Luo, S.; Wang, G.; Peng, Z.; Zeng, W.; Tan, S.; Xi, Y.; Fan, J. Downregulation of tazarotene induced gene-2 (TIG2) in skin squamous cell carcinoma. Eur. J. Dermatol. 2008, 18, 638-641. [CrossRef] [PubMed]

7. Zhao, S.; Li, C.; Ye, Y.-B.; Peng, F.; Chen, Q. Expression of Chemerin Correlates with a Favorable Prognosis in Patients with Non-Small Cell Lung Cancer. Lab. Med. 2011, 42, 553-557. [CrossRef]

8. Pachynski, R.; Zabel, B.A.; Kohrt, H.E.; Tejeda, N.M.; Monnier, J.; Swanson, C.D.; Holzer, A.K.; Gentles, A.J.; Sperinde, G.V.; Edalati, A.; et al. The chemoattractant chemerin suppresses melanoma by recruiting natural killer cell antitumor defenses. J. Exp. Med. 2012, 209, 1427-1435. [CrossRef]

9. Treeck, O.; Buechler, C.; Ortmann, O. Chemerin and Cancer. Int. J. Mol. Sci. 2019, 20, 3750. [CrossRef]

10. Dubois-Vedrenne, I.; Al Delbany, D.; De Henau, O.; Robert, V.; Vernimmen, M.; Langa, F.; Lefort, A.; Libert, F.; Wittamer, V.; Parmentier, M. The antitumoral effects of chemerin are independent from leukocyte recruitment and mediated by inhibition of neoangiogenesis. Oncotarget 2021, 12, 1903-1919. [CrossRef] [PubMed]

11. Bonecchi, R.; Graham, G.J. Atypical Chemokine Receptors and Their Roles in the Resolution of the Inflammatory Response. Front. Immunol. 2016, 7, 224. [CrossRef]

12. Massara, M.; Bonavita, O.; Mantovani, A.; Locati, M.; Bonecchi, R. Atypical chemokine receptors in cancer: Friends or foes? J. Leukoc. Biol. 2016, 99, 927-933. [CrossRef]

13. Sjöberg, E.; Meyrath, M.; Chevigné, A.; Östman, A.; Augsten, M.; Szpakowska, M. The diverse and complex roles of atypical chemokine receptors in cancer: From molecular biology to clinical relevance and therapy. Adv. Cancer Res. 2020, 145, 99-138. [CrossRef]

14. Zabel, B.A.; Nakae, S.; Zúñiga, L.; Kim, J.-Y.; Ohyama, T.; Alt, C.; Pan, J.; Suto, H.; Soler, D.; Allen, S.J.; et al. Mast cell-expressed orphan receptor CCRL2 binds chemerin and is required for optimal induction of IgE-mediated passive cutaneous anaphylaxis. $J$. Exp. Med. 2008, 205, 2207-2220. [CrossRef]

15. Monnier, J.; Lewén, S.; O’Hara, E.; Huang, K.; Tu, H.; Butcher, E.C.; Zabel, B.A. Expression, Regulation, and Function of Atypical Chemerin Receptor CCRL2 on Endothelial Cells. J. Immunol. 2012, 189, 956-967. [CrossRef]

16. Schioppa, T.; Sozio, F.; Barbazza, I.; Scutera, S.; Bosisio, D.; Sozzani, S.; Del Prete, A. Molecular Basis for CCRL2 Regulation of Leukocyte Migration. Front. Cell Dev. Biol. 2020, 8, 615031. [CrossRef] [PubMed]

17. Yin, F.; Xu, Z.; Wang, Z.; Yao, H.; Shen, Z.; Yu, F.; Tang, Y.; Fu, D.; Lin, S.; Lu, G.; et al. Elevated chemokine CC-motif receptor-like 2 (CCRL2) promotes cell migration and invasion in glioblastoma. Biochem. Biophys. Res. Commun. 2012, 429, 168-172. [CrossRef] 
18. Wang, L.-P.; Cao, J.; Zhang, J.; Wang, B.-Y.; Hu, X.-C.; Shao, Z.-M.; Wang, Z.-H.; Ou, Z.-L. The human chemokine receptor CCRL2 suppresses chemotaxis and invasion by blocking CCL2-induced phosphorylation of p38 MAPK in human breast cancer cells. Med. Oncol. 2015, 32, 254. [CrossRef] [PubMed]

19. Akram, I.G.; Georges, R.; Hielscher, T.; Adwan, H.; Berger, M.R. The chemokines CCR1 and CCRL2 have a role in colorectal cancer liver metastasis. Tumor Biol. 2015, 37, 2461-2471. [CrossRef] [PubMed]

20. Del Prete, A.; Sozio, F.; Schioppa, T.; Ponzetta, A.; Vermi, W.; Calza, S.; Bugatti, M.; Salvi, V.; Bernardini, G.; Benvenuti, F.; et al. The Atypical Receptor CCRL2 Is Essential for Lung Cancer Immune Surveillance. Cancer Immunol. Res. 2019, 7, 1775-1788. [CrossRef]

21. Otero, K.; Vecchi, A.; Hirsch, E.; Kearley, J.; Vermi, W.; Prete, A.D.; Gonzalvo-Feo, S.; Garlanda, C.; Azzolino, O.; Salogni, L.; et al. Europe PMC Funders Group Non-redundant role of CCRL2 in lung dendritic cell trafficking. Blood 2012, 116, 2942-2949. [CrossRef] [PubMed]

22. Luangsay, S.; Wittamer, V.; Bondue, B.; De Henau, O.; Rouger, L.; Brait, M.; Franssen, J.-D.; De Nadai, P.; Huaux, F.; Parmentier, M. Mouse ChemR23 Is Expressed in Dendritic Cell Subsets and Macrophages, and Mediates an Anti-Inflammatory Activity of Chemerin in a Lung Disease Model. J. Immunol. 2009, 183, 6489-6499. [CrossRef]

23. Dubois-Vedrenne, I.; De Henau, O.; Robert, V.; Langa, F.; Javary, J.; Al Delbany, D.; Vosters, O.; Canals, E.A.; Vernimmen, M.; Luangsay, S.; et al. Expression of Bioactive Chemerin by Keratinocytes Inhibits Late Stages of Tumor Development in a Chemical Model of Skin Carcinogenesis. Front. Oncol. 2019, 9, 1253. [CrossRef] [PubMed]

24. Danciu, C.; Falamas, A.; Dehelean, C.; Soica, C.; Radeke, H.; Barbu-Tudoran, L.; Bojin, F.; Pînzaru, S.C.; Munteanu, M.F. A characterization of four B16 murine melanoma cell sublines molecular fingerprint and proliferation behavior. Cancer Cell Int. 2013, 13, 75. [CrossRef] [PubMed]

25. Wittamer, V.; Franssen, J.-D.; Vulcano, M.; Mirjolet, J.-F.; Le Poul, E.; Migeotte, I.; Brézillon, S.; Tyldesley, R.; Blanpain, C.; Detheux, M.; et al. Specific Recruitment of Antigen-presenting Cells by Chemerin, a Novel Processed Ligand from Human Inflammatory Fluids. J. Exp. Med. 2003, 198, 977-985. [CrossRef]

26. Bondue, B.; De Henau, O.; Luangsay, S.; Devosse, T.; de Nadai, P.; Springael, J.-Y.; Parmentier, M.; Vosters, O. The Chemerin/ChemR23 System Does Not Affect the Pro-Inflammatory Response of Mouse and Human Macrophages Ex Vivo. PLoS ONE 2012, 7, e40043. [CrossRef]

27. Peyrassol, X.; Laeremans, T.; Gouwy, M.; Lahura, V.; Debulpaep, M.; Van Damme, J.; Steyaert, J.; Parmentier, M.; Langer, I. Development by Genetic Immunization of Monovalent Antibodies (Nanobodies) Behaving as Antagonists of the Human ChemR23 Receptor. J. Immunol. 2016, 196, 2893-2901. [CrossRef]

28. Abel, E.; Angel, J.M.; Kiguchi, K.; DiGiovanni, J. Multi-stage chemical carcinogenesis in mouse skin: Fundamentals and applications. Nat. Protoc. 2009, 4, 1350-1362. [CrossRef] [PubMed]

29. Eden, E.; Navon, R.; Steinfeld, I.; Lipson, D.; Yakhini, Z. GOrilla: A tool for discovery and visualization of enriched GO terms in ranked gene lists. BMC Bioinform. 2009, 10, 48. [CrossRef]

30. Subramanian, A.; Tamayo, P.; Mootha, V.K.; Mukherjee, S.; Ebert, B.L.; Gillette, M.A.; Paulovich, A.; Pomeroy, S.L.; Golub, T.R.; Lander, E.S.; et al. Gene set enrichment analysis: A knowledge-based approach for interpreting genome-wide expression profiles. Proc. Natl. Acad. Sci. USA 2005, 102, 15545-15550. [CrossRef]

31. Chow, M.T.; Luster, A.D. Chemokines in Cancer. Cancer Immunol. Res. 2014, 2, 1125-1131. [CrossRef]

32. Marcuzzi, E.; Angioni, R.; Molon, B.; Calì, B. Chemokines and Chemokine Receptors: Orchestrating Tumor Metastasization. Int. J. Mol. Sci. 2018, 20, 96. [CrossRef]

33. Del Prete, A.; Bonecchi, R.; Vecchi, A.; Mantovani, A.; Sozzani, S. CCRL2, a fringe member of the atypical chemoattractant receptor family. Eur. J. Immunol. 2013, 43, 1418-1422. [CrossRef]

34. Shimada, T.; Matsumoto, M.; Tatsumi, Y.; Kanamaru, A.; Akira, S. A novel lipopolysaccharide inducible C-C chemokine receptor related gene in murine macrophages. FEBS Lett. 1998, 425, 490-494. [CrossRef]

35. Del Prete, A.; Locati, M.; Otero, K.; Riboldi, E.; Mantovani, A.; Vecchi, A.; Sozzani, S. Migration of dendritic cells across blood and lymphatic endothelial barriers. Thromb. Haemost. 2006, 95, 22-28. [CrossRef]

36. Gonzalvo-Feo, S.; Del Prete, A.; Pruenster, M.; Salvi, V.; Wang, L.; Sironi, M.; Bierschenk, S.; Sperandio, M.; Vecchi, A.; Sozzani, S. Endothelial Cell-Derived Chemerin Promotes Dendritic Cell Transmigration. J. Immunol. 2014, 192, 2366-2373. [CrossRef]

37. Del Prete, A.; Muñoz, L.M.; Mazzon, C.; Toffali, L.; Sozio, F.; Za, L.; Bosisio, D.; Gazzurelli, L.; Salvi, V.; Tiberio, L.; et al. The atypical receptor CCRL2 is required for CXCR2-dependent neutrophil recruitment and tissue damage. Blood 2017, 130, 1223-1234. [CrossRef] [PubMed]

38. Mazzotti, C.; Gagliostro, V.; Bosisio, D.; Del Prete, A.; Tiberio, L.; Thelen, M.; Sozzani, S. The Atypical Receptor CCRL2 (C-C Chemokine Receptor-Like 2) Does Not Act as a Decoy Receptor in Endothelial Cells. Front. Immunol. 2017, 8, 1233. [CrossRef]

39. De Henau, O.; DeGroot, G.-N.; Imbault, V.; Robert, V.; De Poorter, C.; Mcheik, S.; Gales, C.; Parmentier, M.; Springael, J.-Y. Signaling Properties of Chemerin Receptors CMKLR1, GPR1 and CCRL2. PLoS ONE 2016, 11, e0164179. [CrossRef] [PubMed]

40. Masiero, M.; Simões, F.; Han, H.D.; Snell, C.; Peterkin, T.; Bridges, E.; Mangala, L.S.; Wu, S.; Pradeep, S.; Li, D.; et al. A Core Human Primary Tumor Angiogenesis Signature Identifies the Endothelial Orphan Receptor ELTD1 as a Key Regulator of Angiogenesis. Cancer Cell 2013, 24, 229-241. [CrossRef] [PubMed]

41. Mazzon, C.; Zanotti, L.; Wang, L.; Del Prete, A.; Fontana, E.; Salvi, V.; Poliani, P.L.; Sozzani, S. CCRL2 regulates M1/M2 polarization during EAE recovery phase. J. Leukoc. Biol. 2016, 99, 1027-1033. [CrossRef] 
42. Reyes, N.; Benedetti, I.; Rebollo, J.; Correa, O.; Geliebter, J. Atypical chemokine receptor CCRL2 is overexpressed in prostate cancer cells. J. Biomed. Res. 2018, 33, 17-23. [CrossRef]

43. Ben Dhaou, C.; Mandi, K.; Frye, M.; Acheampong, A.; Radi, A.; De Becker, B.; Antoine, M.; Baeyens, N.; Wittamer, V.; Parmentier, $\mathrm{M}$. Chemerin regulates normal angiogenesis and hypoxia-driven neovascularization. Angiogenesis 2021, 1-21. [CrossRef]

44. Bozaoglu, K.; Curran, J.E.; Stocker, C.J.; Zaibi, M.S.; Segal, D.; Konstantopoulos, N.; Morrison, S.; Carless, M.; Dyer, T.D.; Cole, S.A.; et al. Chemerin, a Novel Adipokine in the Regulation of Angiogenesis. J. Clin. Endocrinol. Metab. 2010, 95, $2476-2485$. [CrossRef]

45. Kaur, J.; Adya, R.; Tan, B.K.; Chen, J.; Randeva, H.S. Identification of chemerin receptor (ChemR23) in human endothelial cells: Chemerin-induced endothelial angiogenesis. Biochem. Biophys. Res. Commun. 2010, 391, 1762-1768. [CrossRef] [PubMed]

46. Nakamura, N.; Naruse, K.; Kobayashi, Y.; Miyabe, M.; Saiki, T.; Enomoto, A.; Takahashi, M.; Matsubara, T. Chemerin promotes angiogenesis in vivo. Physiol. Rep. 2018, 6, e13962. [CrossRef] [PubMed]

47. Wittamer, V.; Bondue, B.; Guillabert, A.; Vassart, G.; Parmentier, M.; Communi, D. Neutrophil-Mediated Maturation of Chemerin: A Link between Innate and Adaptive Immunity. J. Immunol. 2005, 175, 487-493. [CrossRef] [PubMed] 